\title{
Efficient Design Of Biological Experiments For Dose-Response Modeling In Nanomaterial Toxicology Studies
}

Kai Wang

West Virginia University

Follow this and additional works at: https://researchrepository.wvu.edu/etd

\section{Recommended Citation}

Wang, Kai, "Efficient Design Of Biological Experiments For Dose-Response Modeling In Nanomaterial Toxicology Studies" (2011). Graduate Theses, Dissertations, and Problem Reports. 483.

https://researchrepository.wvu.edu/etd/483

This Thesis is protected by copyright and/or related rights. It has been brought to you by the The Research Repository @ WVU with permission from the rights-holder(s). You are free to use this Thesis in any way that is permitted by the copyright and related rights legislation that applies to your use. For other uses you must obtain permission from the rights-holder(s) directly, unless additional rights are indicated by a Creative Commons license in the record and/ or on the work itself. This Thesis has been accepted for inclusion in WVU Graduate Theses, Dissertations, and Problem Reports collection by an authorized administrator of The Research Repository @ WVU. For more information, please contact researchrepository@mail.wvu.edu. 


\title{
Efficient Design Of Biological Experiments For Dose-Response \\ Modeling In Nanomaterial Toxicology Studies
}

\author{
Kai Wang \\ Thesis submitted to the \\ College of Engineering and Mineral Resources \\ at West Virginia University \\ in partial fulfillment of the requirements \\ for the degree of \\ Master of Science \\ in \\ Industrial Engineering \\ Feng Yang, Ph.D., Chair \\ Robert Creese, Ph.D. \\ Majid Jaridi, Ph.D. \\ Department of Industrial and Management Systems Engineering \\ Morgantown, West Virginia \\ 2011
}

Keywords: Multiple objective genetic algorithm, A-optimum, D-optimum,Heterosedastic Model, Biological experiment, Bayesian procedure Copyright 2011 Kai Wang 


\begin{abstract}
Efficient Design Of Biological Experiments For Dose-Response

Modeling In Nanomaterial Toxicology Studies
\end{abstract}

Kai Wang

This thesis is concerned with developing two-stage Bayesian optimum design procedure in nanotoxicology with simulation experiments. The proposed design method adopts suitable nonlinear dose response curve and non-constant variance model in experimental modeling, which more adequately represents the desirable properties of bioassay-experiment. A multiple objective genetic algorithm were used to select best set of non-dominated experiments on Pareto front. The proposed approach has been shown to provide better design than traditional experiment method in terms of D-optimum design and A-optimum design. 


\section{ACKNOWLEDGMENTS}

I would like to express my thanks to my advisor, Dr. Feng Yang, for her great guidance and continuous support throughout this research. I am also thankful to Dr. Robert Creese and Dr. Majid Jaridi for serving on my committee, and for their insightful comments and suggestions in preparing this thesis.

Finally, I would like to thank my parents for their selfless love. 


\section{Contents}

List of Abbreviations $\quad$ vi

List of Figures

List of Tables $\quad$ viii

1 Introduction $\quad 1$

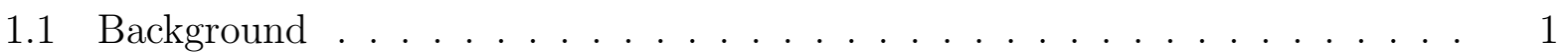

1.2 Objectives and methodology . . . . . . . . . . . . . . . 2

2 Literature Review $\quad 3$

3 Methodology $\quad 6$

3.1 Formalization of Prior Information . . . . . . . . . . . . . . 7

3.1.1 The Heteroscedastic Dose-Response Model . . . . . . . . . . . . . . . 7

3.1.2 Estimation of the Dose-Response Model . . . . . . . . . . . . . . . 9

3.2 Multi-Objective Optimum Design . . . . . . . . . . . . . . . . 12

3.2.1 Design Criteria . . . . . . . . . . . . . . . . 13

3.2.2 The Multi-Objective Optimization Methods . . . . . . . . . . . 16

4 Empirical Study $\quad 20$

4.1 Case 1: Nonlinear model with increasing variance . . . . . . . . . . . . 20

4.1.1 First-stage Experimentation and Modeling . . . . . . . . . . . . . . 21

4.1.2 Second-stage Design of Experiments . . . . . . . . . . . . . . . 22 
4.1.3 Design Comparison . . . . . . . . . . . . . . . . 24

4.2 Case 2: Nonlinear model with bell shape variance . . . . . . . . . . . . . 24

4.2.1 First-stage Experimentation and Modeling . . . . . . . . . . . 25

4.2.2 Second-stage Design of Experiments . . . . . . . . . . . . . . 27

4.2 .3 Design Comparison . . . . . . . . . . . . . . . . . . . 27

5 Conclusions and future study $\quad 30$

A Result of Empirical Study 33

$\begin{array}{ll}\text { References } & 36\end{array}$ 


\section{List of Abbreviations}

$B M D$ benchmark dose

$B M R$ benchmark response

NOAEL no observed adverse effect level 


\section{List of Figures}

2.1 An example of dose-response data. . . . . . . . . . . . . . . . . . . 4

3.1 The two-stage Bayesian framework for experimental design. . . . . . . . . . 7

3.2 Dose-response data for $\mathrm{TiO}_{2}$ nanowires. . . . . . . . . . . . . . . . 9

4.1 Initial design for case $1 \ldots \ldots \ldots$. . . . . . . . . . . . . . . . . . . 21

4.2 Optimal Pareto front of two-stage design procedure for case 1 . . . . . . . . . 23

4.3 Initial design for case $2 \ldots \ldots \ldots \ldots$

4.4 Optimal Pareto front of two-stage design procedure for case $2 . \quad$. . . . . . . 28 


\section{List of Tables}

4.1 Result of traditional design for case 1. . . . . . . . . . . . . . . . . 24

4.2 Part of optimal result of proposed two-stage design procedure for case $1 . \quad$. . 25

4.3 Result of traditional design for case $2 \ldots \ldots$. . . . . . . . . . . . . . . . . 28

4.4 Part of optimal result of proposed two-stage design procedure for case $2 . \quad$. . 29

A.1 Optimal result of proposed two-stage design procedure for case 1. . . . . . . 34

A.2 Optimal result of proposed two-stage design procedure for case $2 . \quad$. . . . . 35 


\section{Chapter 1}

\section{Introduction}

\section{$1.1 \quad$ Background}

With the advancement of nanotechnology in a wide range of applications many nanometersized particles-" nanoparticles"-are now commercially available. However, the nanomaterial risk assessment lags far behind its development, and remains an urgent need [1]. One of the most fundamental steps in assessing the risk of a nanomaterial is to understand and properly characterize its dose-response relationship $[2,3]$. To estimate such relationships, biological animal experiments need to be performed at different dose levels to observe the corresponding bioactivity responses of animals. Because of costs, ethics, or other limitations on resources or time, sample sizes are usually restricted and efficient use of available resources is critical. Thus, the design of experiments, i.e., the selection of experimental doses and the allocation of animals, plays an important role in the success of dose-response modeling.

Currently, the designs used in nanotoxicology studies are mainly selected by experimenters based on empirical experiences in a somewhat arbitrary manner $[4,5]$. Such designs, which is referred to as the traditional design, usually involve equally-spaced doses on a linear or log scale and equal allocation of animals, and may be far from optimum for dose-response modeling due to the following two major reasons. First, the dose-response curves may well be nonlinear [6-8], and thus the optimum design of experiments depends on the specific functional model representing the target curve $[9,10]$. Second, with constrained resources, typically the amount of data allowed to be collected are not only relatively scarce and highly variable, but almost certainly possess different variability across the dose range being inves- 
tigated [11]; this variance heterogeneity strongly suggests against the traditional design [1217], and the choice of a good design is very important to model estimation. Therefore, there is a great need to have a design of experiments method which is able to utilize the limited resources in the best possible way in dose-response studies.

\subsection{Objectives and methodology}

The objective of the proposed research is to develop an experimental design procedure, which accommodates the nonlinear nature of dose-response curves and variance heterogeneity of toxicity data, to guide the dose selection and animal allocation in biological experiments for the efficient generation of dose-response relationships. The proposed design procedure has been built in a two-stage Bayesian paradigm [18], which provides a statistically valid mechanism to utilize prior information for the design of future experiments. Most suitable dose-response as well as variance models has been identified to describe the toxicity data. Bootstrapping, a computationally intensive resampling method as opposed to conventional statistical inference methods, was used to derive important information from the preliminary data required by the subsequent experimental design. To achieve practically useful designs, multiple design criteria was considered simultaneously, and multi-objective metaheuristics was adapted to search for a set of Pareto optimum designs [19], which allow for the evaluation of various trade-offs in practical experimental settings.

The outcome of this thesis has resulted in an efficient experimental design procedure which is able to characterize substances by high-quality dose-response profiles using less experiments. The methods obtained from the proposed work has substantially reduced the experimental cost and time in toxicology studies, alleviate the rising concerns for animal ethics [20, 21], and accelerate the progress toward quantifying the risk, safety and health effects of environmental and occupational exposure to nanomaterials. 


\section{Chapter 2}

\section{Literature Review}

Estimation of the dose-response relationships is a prerequisite for quantifying potential risks from exposure to nanomaterials, as well as a starting point for safety evaluation and guideline/standard setting. Prediction of risks at a given or expected exposure level can only be performed with a well established dose-response curve representing the toxicological profile of a substance. The curve also allows for the prediction of exposures at responses

of different severities (e.g., the BMD), which assists the risk assessor to make judgments to protect a population from increasingly severe effects.

However, in the existing nanotoxicology studies [5, 22], complete characteristic profiles have been rarely obtained due to the scarcity and high variability of toxicity data collected via costly and time-consuming experiments. In those works, typically experiments were carried out at four or five doses with equal number of samples at each dose, and multiple comparison methods $[23,24]$ were used to address questions as: Which of the selected doses are (relevantly) different from control? What dose levels may cause harmful effects? Such a multiple comparison analysis along with the NOAEL (no observed adverse effect level) derived from it, though provides valuable information, is not adequate for risk quantification since inference is confined to experimental doses [25-28]. In contrast, a dose-response profile allows for the estimation of responses over a wide range of doses, and is also able to provide an estimate for the BMD, the dose that corresponds to a specified level of additional response. The BMD is considered as a promising alternative over the traditional NOAEL-conditional on the acquirement of high-quality dose-response models, which is difficult and a major 
hindrance in using the BMD approach in risk assessment. Therefore, it is important to efficiently design experiments so that nanomaterials can be adequately characterized by their dose-response profiles using limited resources available.

Experimental design for efficient dose-response modeling is difficult. Figure 2.1, which is generated from the data in [29], provides an example of dose-response data along with the fitted curve. As depicted in the graph, the dependence of response upon dose levels follows a nonlinear pattern and the variance of the responses varies substantially over the dose arrange. To achieve efficiency in dose-response studies, the experimental design questions that need to be addressed are: At what dose levels should the experiments be performed and how many animals should be allocated to each dose level? The nonlinear nature of the curve and the heteroscedasticity of the data make it particularly challenging to efficiently design experiments.

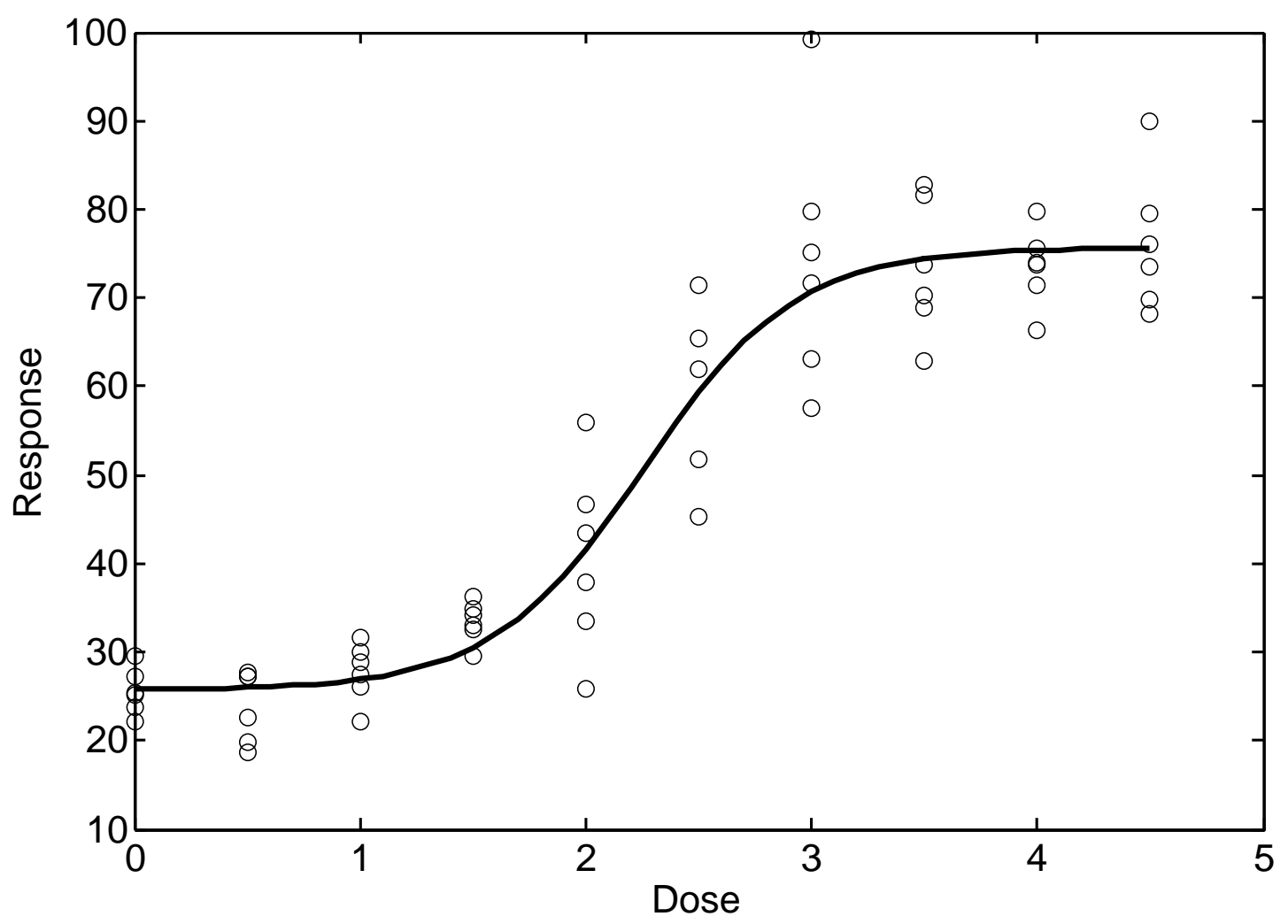

Figure 2.1: An example of dose-response data. 
As already pointed out, the traditional way of designing experiments in the vast majority of nanotoxicology studies may well lead to the inefficient use of resources. Some researchers $[2,30-33]$ recognized the nonlinearity of dose-response relationships, and resorted to standard optimum design techniques [9] to approach the design of experiments in dose-response studies. These works have relied on the assumption of constantly variance throughout the dose range, which typically does not hold in reality. There is also a small body of literature by statisticians who investigated the optimum design for statistical modeling in general while taking into account the existence of variance heterogeneity. In this stream of work [34-44], the authors usually had a particular interest in pursuing analytical solutions under various simplistic assumptions. For instance, Schulz and Endrenyi [34] found the optimal designs for two nonlinear models when the error variance is a power of the mean; DasGupta et al. [35] derived the optimal designs under a normal prior for the simple linear model when the error variance is a power function or an exponential function of the mean. These results, though theoretically appealing, are often impractical for toxicology studies where the required assumptions no longer hold.

In light of this, it is the intention of the proposed work to develop an experimental design procedure for efficient dose-response modeling while adequately considering the nonlinearity of the underlying curve, the variance heterogeneity at different dose levels, and the special features and practical constraints of biological experiments. 


\section{Chapter 3}

\section{Methodology}

In dose-response modeling for toxicology studies, the experimental design is concerned with determining the dose locations and animal allocations. The optimum design depends on the nonlinear dose-response curve and the variance structure over the dose range, which are both unknown when designing experiments with data yet to be collected. To circumvent this problem, a Bayesian approach which provides a coherent framework for the design of biological experiments is proposed.

Prior to experimentation, pertinent information on the unknown quantities that may affect the optimum design is usually available. Such prior information can be obtained from earlier related experiments, observational studies, or subjective beliefs from personal observations. As a matter of fact, it is typical for biologists to perform a small number of exploratory experiments before launching the relatively large-scale and more informative experimentation, and the former are usually used to assist the design of the latter. The Bayesian approach simply provides a statistically valid mechanism to utilize the information obtained from the earlier experiments for the design of later ones. In light of this, we propose a Bayesian experimental design procedure consisting of two stages of experimentation. And a high-level description of the procedure is given in Figure 3.1.

As shown in Figure 3.1, in the first stage, useful information for subsequent experimental design was derived from a small amount of preliminary data, the specifics of which are given in Section 3.1. In the second stage of the Bayesian framework, the derived prior information has been used to find the approximate optimum design of experiments, which is 


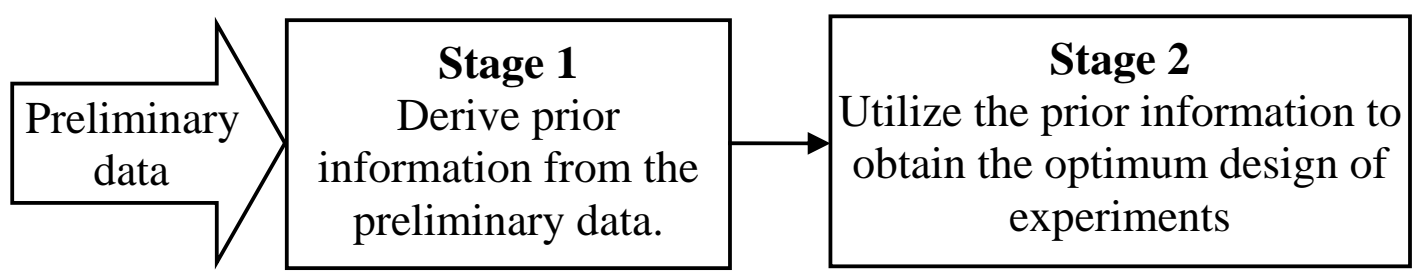

Figure 3.1: The two-stage Bayesian framework for experimental design.

detailed in Section 3.2. The two-stage design procedure then was thoroughly evaluated via simulation experiments (Chapter 4). Lastly, to illustrate its use in toxicology, the proposed design procedure has been applied to guide the design of experiments in the pulmonary toxicity study of $\mathrm{TiO}_{2}$ nanoparticles.

\subsection{Formalization of Prior Information}

To obtain a most informative Bayesian design, it is important to incorporate all available prior information. Dickey and Garthwaite [45] developed useful methods for elicitation and quantification of an expert's opinion, and Kadane [46] considered the practical issues in subjective elicitation for clinical trials. Related data available in the literature is also a valuable source of information $[18,47]$. In this work, it is assumed that in the initial stage, a small amount of experiments are performed (likely following the traditional naive design), and they can provide useful information regarding the underlying dose-response curve and the variance structure of the data, which are needed for the second-stage optimum design. In the next section, we present the methods used to extract these information from the initial data set.

\subsubsection{The Heteroscedastic Dose-Response Model}

Here, we illustrate the proposed methods via continuous dose-response models, and for models with quantal responses, the methods can be adapted straightforwardly. Let $Y$ be the random response of a biological experiment. We denote a dose-response curve by the general 
differentiable functional model

$$
\mathrm{E}[Y \mid x]=f(x, \boldsymbol{\theta})
$$

where $x$ represents the dose level and $\boldsymbol{\theta}$ the $p \times 1$ vector of unknown parameters. The random variable $Y$ is related to its mean by the relationship

$$
Y=\mathrm{E}[Y \mid x]+\epsilon
$$

where $\epsilon$ is the error term. It is assumed that we have independent error at different values of $x$, and that $\epsilon$ is normally distributed with mean zero and dose-dependent variance given as follows

$$
\operatorname{Var}[\epsilon \mid x]=g(x, \gamma)
$$

where $\gamma$ represents the unknown $q \times 1$ parameter vector.

\section{Candidate models for dose-response curves}

For a nanomaterial, the dependence of a selected biological response on the dose may follow a range of patterns [8] such as linear, exponential, logistic, etc. For the modeling of the dose-response curve, the most suitable functional form needs to be selected. In this work, six different mathematical forms as shown below are considered as the potential candidates for the dose-response model $f(x, \boldsymbol{\theta})$ :

- Linear: $f(x, \boldsymbol{\theta})=\theta_{0}+\theta_{1} x$

- Quadratic: $f(x, \boldsymbol{\theta})=\theta_{0}+\theta_{1} x+\theta_{2} x^{2}$

- Linear log-dose: $f(x, \boldsymbol{\theta})=\theta_{0}+\theta_{1} \log (x+1)$

- Exponential: $f(x, \boldsymbol{\theta})=\theta_{0}+\theta_{1} \exp \left(x / \theta_{2}\right)$

- $\mathrm{E}_{\text {max }}: f(x, \boldsymbol{\theta})=\theta_{0}+\theta_{1} x /\left(\theta_{2}+x\right)$

- Logistic: $f(x, \boldsymbol{\theta})=\theta_{0}+\theta_{1} /\left(1+\exp \left(\left(\theta_{2}-x\right) / \theta_{3}\right)\right)$. 
These models represent the most frequently encountered dose-response shapes [3] and are expected to provide an adequate candidate pool for the relationship being investigated.

\section{Dose-dependent variance model}

The variance of the error $\epsilon$ is dependent on the dose level $x$ as shown in model (3.3). We assume that the variance model $g(x, \gamma)$ can be described by one of the following forms:

$$
\begin{aligned}
& g_{1}(x, \gamma)=\gamma_{1} \exp \left(-\gamma_{2} x\right), \quad \gamma_{1}>0 \\
& g_{2}(x, \gamma)=x^{\gamma_{1}}(b-x)^{\gamma_{2}}, \quad \gamma_{1} \geq 0 \text { and } \gamma_{2} \geq 0
\end{aligned}
$$

with $\gamma=\left(\gamma_{1}, \gamma_{2}\right)$ being the unknown parameters. These two models are very flexible and useful in practice (e.g. [39], [48]), and are expected to adequately describe the various variance structures in toxicity data. For instance, $g_{2}(x, \gamma)$ is able to capture the variance trend for the data in Figure 2.1, and $g_{1}(x, \gamma)$ for the data in Figure 3.2.

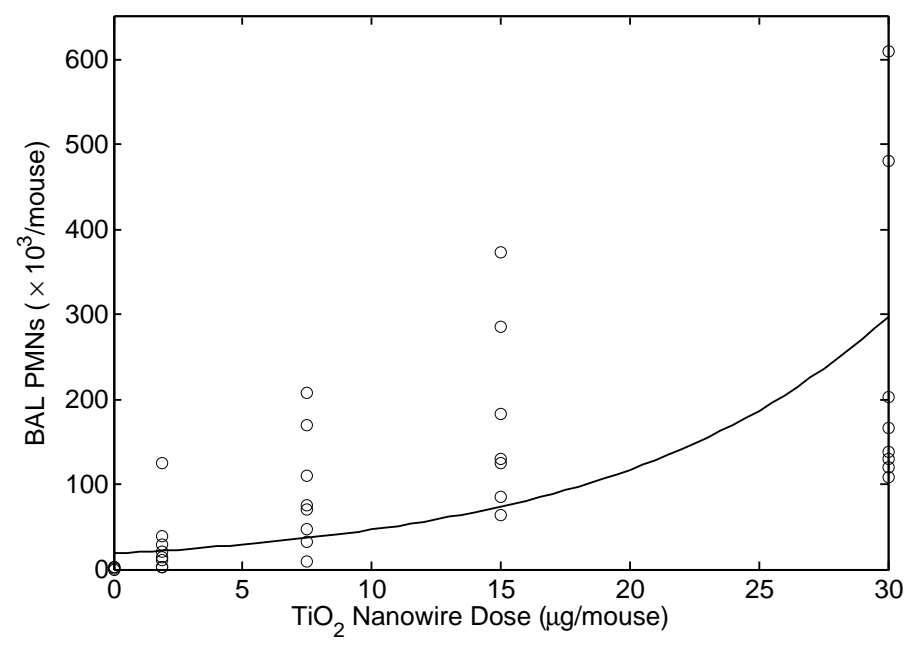

Figure 3.2: Dose-response data for $\mathrm{TiO}_{2}$ nanowires.

\subsubsection{Estimation of the Dose-Response Model}

At this first stage of Bayesian framework (Figure 3.1), a small amount of preliminary data has been collected which serves two major purposes: one is to help determine the dose 
range of interest, say $\left[x_{L}, x_{U}\right]$, and the other is to provide information for the design of the next-stage experimentation. The latter is the primary concern in this part, and the useful information considered here includes the approximate dose response model, the distribution of the model parameters, and the variance structure involved.

\section{Model selection and estimation}

Given the preliminary data, the first step is to estimate the variance model if variance heterogeneity exists. Tests for the presence of data heterogeneity are given in [40, 49-51]. One of the two variance models presented above can be easily selected by visualizing the data, and the selected model was fitted using nonlinear least-square methods. We denote the fitted variance structure model as $g(x, \widehat{\gamma})$. As an approximation, $g(x, \widehat{\gamma})$ is assumed to be the true variance model once obtained and was used in the remaining of the Bayesian design process.

After establishing the variance model $g(x, \widehat{\gamma})$ of the data, the six dose-response candidate models, which were introduced in Section 3.1.1, were fitted to the data using the weighted least squares methods. Note that the weighted least squares is particularly developed to perform heteroscedastic nonlinear regressions. Among the six model candidates, the one that leads to the smallest squared error in model fitting was selected to approximate the underlying dose-response curve. We denote the selected dose-response model as $f(x, \widehat{\boldsymbol{\theta}})$, with $\widehat{\boldsymbol{\theta}}$ being the vector of estimated parameters.

\section{Quantifying the uncertainty of model estimates}

With the fitted dose-response model $f(x, \widehat{\boldsymbol{\theta}})$, we next seek to quantify the distribution of the model parameters because they cannot be known with absolute certainty especially at this early stage of experimentation. Since the conventional statistical inference relies on the large-sample theory [52] which may well not apply to the initial data here, we propose to use the bootstrap method, a computationally-intensive resampling method, to derive the 
distribution of the model estimates. The bootstrap method is presented in Efron et al. [53] and Davison et al. [54], and Slob et al. [55] exemplifies its application in toxicology studies. The basic idea of bootstrap is that a large number of artificial data sets can be generated by Monte Carlo simulation [56], and each data set leads to a point estimate for the quantity of interest, $\boldsymbol{\theta}$. Thus, based on the large number of estimates, the distribution of $\boldsymbol{\theta}$, denoted as $\Pi(\boldsymbol{\theta})$, can be derived. For regression models, wild bootstrap and pair bootstrap are two most popular methods robust to heteroskedasticity of unknown variance function for inference.

Efron [57] confirms that pair bootstrap, first proposed by Freedman [58] and later modified by Mammen [59] and Flachaire [60], can give trustworthy estimate of coefficient of nonlinear model, $\hat{\boldsymbol{\theta}}$, even if the error is not constant. The other technique used to overcome heteroskedasticity is the so-called wild bootstrap, developed by Liu [61], following suggestions by $\mathrm{Wu}$ [62], who points out that bootstrap the residuals, basing on the assumption of the exchangeability of the errors, is not robust against heteroscedasticity of error variance and suggests that residual should be revised before bootstrap. Recently, Davidson et al. [54] proposes a procedure, which uses Rademacher distribution to generate the distribution of $\varepsilon_{t}^{*}$ and gives exact results for some specific cases. Both pair bootstrap and wild bootstrap can provide consistent inference with heteroskedasticity, some author such as Valletta et al.and MacKinnon $[63,64]$, however, believe that wild bootstrap generally may provide more accurate distribution, since, unlike pair bootstrap, it imposes a restriction on each bootstrap sample. Horowitz [65] gives supporting simulation evidence to Mammen's [66] proposal that the distribution of the wild bootstrap converges to the correct sampling distribution faster than the pair bootstrap.

Procedure for wild bootstrap for heterosedastic model is given as follows [54]:

1. Fit nonlinear model $f(x, \boldsymbol{\theta})$ with ordinary least squares, then compute the corresponding predicted $\hat{y}_{i}$ from the fitted model $f\left(x_{i}, \hat{\boldsymbol{\theta}}\right)$.

2. Compute modified residuals $r_{i}=\frac{v_{i}^{1 / 2}\left(y_{i}-\hat{y}_{i}\right)}{\left(1-h_{i}\right)^{1 / 2}}$ where $v_{i}$ is defined as $\operatorname{Var}^{-1}\left(x_{i}\right)$, and $h_{i}$ is leverage value, which is on the diagonal of matrix $\mathbf{J}\left(\mathbf{J}^{T} \mathbf{V} \mathbf{J}\right)^{-1} \mathbf{J}^{T} \mathbf{V}$, in which $\mathbf{J}$ is 
the row vector of partial derivative of $f(x, \boldsymbol{\theta})$ with respect to $\boldsymbol{\theta}$ and $\mathbf{V}$ is the diagonal matrix of weights $v_{i}$.

3. Compute the centered modified residual $r_{i}-\bar{r}$.

4. Take a sample of the $\mathrm{n}$ modified and centered residuals $\varepsilon_{i}^{*}$ from Rademacher distribution $[67]$

$$
\operatorname{Pr}\left\{\varepsilon_{i}^{*}=-\left(r_{i}-\bar{r}\right)\right\}=\frac{1}{2} \quad \operatorname{Pr}\left\{\varepsilon_{i}^{*}=\left(r_{i}-\bar{r}\right)\right\}=\frac{1}{2},
$$

where $*$ means the simulated copies sampled by taking with replacement, which is same in the following of this thesis.

5. Set $x_{i}^{*}=x_{i}$ and $y_{i}^{*}=\hat{y}_{i}+v_{i}^{-\frac{1}{2}} \varepsilon_{i}^{*}, i=1,2, \ldots, n$, and fit the nonlinear model $f\left(x_{i}, \hat{\boldsymbol{\theta}}\right)$ by weighted least squares with weight set $v_{i}$ to obtain parameter estimates for the sampled data $\left(\mathbf{X}^{*}, \mathbf{Y}^{*}\right)$.

6. Repeat steps 4 and 5 for $R$ (e.g., $R=999$ ) times to generate $R$ sets of parameter estimates $\hat{\boldsymbol{\theta}}_{1}, \hat{\boldsymbol{\theta}}_{2}, \ldots, \hat{\boldsymbol{\theta}}_{R}$.

As a result, the prior information derived at the first stage of the Bayesian diagram

are: the dose response model $f(x, \widehat{\boldsymbol{\theta}})$, the mean and distribution of the model parameter $\Pi(\boldsymbol{\theta})$ with wild bootstrap, and the variance model $g(x, \widehat{\gamma})$. Note that the variance parameter $\gamma$ is assumed known as $\hat{\gamma}$, and the uncertainty of $\gamma$ is not considered in this work.

\subsection{Multi-Objective Optimum Design}

At the second stage of the Bayesian diagram (Figure 3.1), we utilize the information obtained from the previous stage, and seek to find a design for the subsequent experiments that best meets the multiple goals that the researchers may have. In toxicology studies of nanomaterials, the most important goals include, but are not limited to, achieving the most precise estimates for (i) the dose-response model and (ii) the BMD. However, in the 
experimental design literature, multiple goals have been barely considered simultaneously. Here, we has formulated the optimum design as a multi-objective optimization problem, and adapt a metaheuristic algorithm to search for the approximate optimum designs.

Suppose that the second-stage experimentation allows for a pre-specified, limited number of $N$ samples to be collected. A design is specified as

$$
\eta_{d}=\left\{\left(x_{i}, w_{i}\right) ; i=1,2, \ldots, d\right\}, \quad \text { with } \sum_{i=1}^{d} w_{i}=1
$$

The number of distinct dose levels is denoted as $d$. In this work, following the recommendation of Kuljus et al. [32], we set $d$ as 4 or 5 based on the shape of the dose-response curve, which has been roughly estimated in the previous stage. Let $n_{i}(i=1,2, \ldots, d)$ be the number of samples allocated to dose $x_{i}$, and $w_{i}=n_{i} / N$ represents the proportion of samples at $x_{i}$. The task here is to find the design $\eta_{d}$ that excels in terms of the two criteria (Section 3.2.1), which corresponds to the two goals introduced below.

\subsubsection{Design Criteria}

We derive the mathematical expressions of the two criteria of interest: the D-optimality crite-

rion, which measures the precision of the estimated parameters $\widehat{\boldsymbol{\theta}}$ in the dose-response model, and the variance of the BMD estimate, which quantifies how precise the BMD estimate is.

\section{Information matrix per observation}

Under the assumption of independent normal errors in (3.2), the kernel of the likelihood function for an observation $y$ made at $x$ is given as

$$
L(y, x, \boldsymbol{\theta}, \boldsymbol{\gamma})=\frac{1}{\sqrt{g(x, \boldsymbol{\gamma})}} \exp \left(-\frac{1}{2} \frac{(y-f(x, \boldsymbol{\theta}))^{2}}{g(x, \boldsymbol{\gamma})}\right)
$$


Thus, the logarithm of the kernel of the likelihood function is

$$
\ell(y, x, \boldsymbol{\theta}, \boldsymbol{\gamma})=-\frac{1}{2}\left(\log g(x, \boldsymbol{\gamma})+\frac{1}{g(x, \boldsymbol{\gamma})}(y-f(x, \boldsymbol{\theta}))^{2}\right)
$$

The information matrix per observation on the parameters $(\boldsymbol{\theta}, \boldsymbol{\gamma})$ is defined as

$$
\mathbf{I}(x, \boldsymbol{\theta}, \boldsymbol{\gamma})=\left[\begin{array}{cc}
\mathbf{I}_{\boldsymbol{\theta}} & \mathbf{I}_{\boldsymbol{\theta} \boldsymbol{\gamma}} \\
\mathbf{I}_{\boldsymbol{\gamma} \boldsymbol{\theta}} & \mathbf{I}_{\boldsymbol{\gamma}}
\end{array}\right]=\left[\begin{array}{cc}
-\mathrm{E}\left[\partial^{2} \ell / \partial \boldsymbol{\theta}^{2}\right] & -\mathrm{E}\left[\partial^{2} \ell / \partial \boldsymbol{\theta} \partial \boldsymbol{\gamma}\right] \\
-\mathrm{E}\left[\partial^{2} \ell / \partial \boldsymbol{\gamma} \partial \boldsymbol{\theta}\right] & -\mathrm{E}\left[\partial^{2} \ell / \partial \boldsymbol{\gamma}^{2}\right]
\end{array}\right]
$$

Substituting the expression of the likelihood function (3.8) into (3.9) yields the information matrix as follows. The $(k, j)^{t h}$ element of the block $\mathbf{I}_{\boldsymbol{\theta}}$ is:

$$
-\mathrm{E}\left[\frac{\partial^{2} \ell}{\partial \theta_{k} \partial \theta_{j}}\right]=\frac{1}{g(x, \boldsymbol{\gamma})} \frac{\partial f(x, \boldsymbol{\theta})}{\partial \theta_{k}} \frac{\partial f(x, \boldsymbol{\theta})}{\partial \theta_{j}}
$$

The $(k, j)^{t h}$ element of the block $\mathbf{I}_{\boldsymbol{\gamma}}$ is:

$$
-\mathrm{E}\left[\frac{\partial^{2} \ell}{\partial \gamma_{k} \partial \gamma_{j}}\right]=\frac{1}{2[g(x, \gamma)]^{2}} \frac{\partial g(x, \gamma)}{\partial \gamma_{k}} \frac{\partial g(x, \gamma)}{\partial \gamma_{j}}
$$

Both $\mathbf{I}_{\boldsymbol{\theta} \boldsymbol{\gamma}}$ and $\mathbf{I}_{\boldsymbol{\gamma} \boldsymbol{\theta}}$ are zero matrices.

Since the information per observation is given by $\mathbf{I}(x, \boldsymbol{\theta}, \boldsymbol{\gamma})$, the Fisher information matrix for the $N$ observations obtained at $d$ dose levels is $\sum_{i=1}^{d} n_{i} \mathbf{I}\left(x_{i}, \boldsymbol{\theta}, \boldsymbol{\gamma}\right)$. Standardized by the sample size $N$, the information matrix for the design $\eta_{d}=\left\{\left(x_{i}, w_{i}\right) ; i=1,2, \ldots, d\right\}$ equals

$$
\mathbf{I}\left(\eta_{d}, \boldsymbol{\theta}, \gamma\right)=\sum_{i=1}^{d} w_{i} \mathbf{I}\left(x_{i}, \boldsymbol{\theta}, \gamma\right)
$$

The two design criteria considered here are functions of the information matrix (3.12), and are specified below. 


\section{The D-optimality criterion}

D-optimality is one of the most popular design strategies [9] and it seeks to maximize determinant of this information matrix. For normally distributed errors, this strategy is equivalent to minimizing the volume of the confidence ellipsoid for the parameters of interest $\boldsymbol{\theta}$. Mathematically, the Bayesian D-optimality criterion is defined as

$$
\mathrm{E}_{\boldsymbol{\theta}}\left|\mathbf{I}\left(\eta_{d}, \boldsymbol{\theta}, \boldsymbol{\gamma}\right)\right|=\int_{\boldsymbol{\theta}}\left|\mathbf{I}\left(\eta_{d}, \boldsymbol{\theta}, \boldsymbol{\gamma}\right)\right| d \Pi(\boldsymbol{\theta})
$$

\section{The precision criterion for the BMD estimate}

In toxicology studies, BMD is the dose level of particular interest. To achieve a precise estimate for the BMD, we use the variance of the BMD estimate as our second criterion.

BMD is the dose that corresponds to a specified level of additional response called the benchmark response (BMR), and the BMR must be determined before calculating the BMD. For a continuous endpoint, BMR can be defined in different ways [26], and we take the relative definition for an example: $\mathrm{BMR}=(f(\mathrm{BMD}, \boldsymbol{\theta})-f(0, \boldsymbol{\theta})) / f(0, \boldsymbol{\theta})$. For a predetermined BMR, the BMD is calculated as follows

$$
\mathrm{BMD}=f^{-1}(\mathrm{BMR} \cdot f(0, \boldsymbol{\theta})+f(0, \boldsymbol{\theta}), \boldsymbol{\theta}),
$$

and the variance of the BMD estimate is:

$$
\begin{aligned}
\operatorname{Var}(B \hat{M} D) & =\left(\frac{\partial f(\mathrm{BMD}, \boldsymbol{\theta})}{\partial \boldsymbol{\theta}}\right)^{T}\left(\left(\frac{\partial f(x, \boldsymbol{\theta})}{\partial \boldsymbol{\theta}}\right)^{T} \mathbf{W}\left(\frac{\partial f(x, \boldsymbol{\theta})}{\partial \boldsymbol{\theta}}\right)\right)^{-1}\left(\frac{\partial f(\mathrm{BMD}, \boldsymbol{\theta})}{\partial \boldsymbol{\theta}}\right) \\
& =\frac{1}{N}\left(\frac{\partial f(\mathrm{BMD}, \boldsymbol{\theta})}{\partial \boldsymbol{\theta}}\right)^{T} \mathbf{I}^{-1}\left(\eta_{d}, \boldsymbol{\theta}\right)\left(\frac{\partial f(\mathrm{BMD}, \boldsymbol{\theta})}{\partial \boldsymbol{\theta}}\right) \\
& =\frac{1}{N} \operatorname{tr}\left(\frac{\partial f(\mathrm{BMD}, \boldsymbol{\theta})}{\partial \boldsymbol{\theta}}\right)\left(\frac{\partial f(\mathrm{BMD}, \boldsymbol{\theta})}{\partial \boldsymbol{\theta}}\right)^{T} \mathbf{I}^{-1}\left(\eta_{d}, \boldsymbol{\theta}\right)
\end{aligned}
$$


with $\partial f(\mathrm{BMD}, \boldsymbol{\theta}) / \partial \boldsymbol{\theta}$ being the derivative vector with respect to $\boldsymbol{\theta}$. The Bayesian variance of the BMD estimate, i.e., our second design criterion, is expressed as:

$$
\mathrm{E}_{\boldsymbol{\theta}}\left[\operatorname{tr} \mathbf{A} \mathbf{I}^{-1}\left(\eta_{d}, \boldsymbol{\theta}\right)\right]=\int_{\boldsymbol{\theta}} \operatorname{tr} \mathbf{A} \mathbf{I}^{-1}\left(\eta_{d}, \boldsymbol{\theta}\right) d \Pi(\boldsymbol{\theta}) .
$$

Where $\mathbf{I}\left(\eta_{d}, \boldsymbol{\theta}\right)$ is given as

$$
\mathbf{I}\left(\eta_{d}, \boldsymbol{\theta}\right)=\sum_{i=1}^{d} w_{i} \mathbf{I}\left(x_{i}, \boldsymbol{\theta}\right)
$$

which is the same as $\mathbf{I}_{\boldsymbol{\theta}}$ defined in (3.10). A is defined as

$$
\mathbf{A}=\left(\frac{\partial f(\mathrm{BMD}, \boldsymbol{\theta})}{\partial \boldsymbol{\theta}}\right)\left(\frac{\partial f(\mathrm{BMD}, \boldsymbol{\theta})}{\partial \boldsymbol{\theta}}\right)^{T}
$$

\subsubsection{The Multi-Objective Optimization Methods}

We formulate the optimum design as the multi-objective optimization problem with two goals:

$$
\begin{aligned}
\text { Objective 1: } & \max _{\eta_{d}} \mathrm{E}_{\boldsymbol{\theta}}\left|\mathbf{I}\left(\eta_{d}, \boldsymbol{\theta}, \boldsymbol{\gamma}\right)\right| \\
\text { Objective 2: } & \min _{\eta_{d}} \mathrm{E}_{\boldsymbol{\theta}}\left[\operatorname{tr} \mathbf{A I}_{\boldsymbol{\theta}}^{-1}\right] \\
\text { With } & \eta_{d}=\left\{\left(x_{i}, w_{i}\right) ; i=1,2, \ldots, d\right\} \\
\text { Subject to: } & x_{i} \in\left[x_{L}, x_{U}\right], \text { and } \sum_{i=1}^{d} w_{i}=1
\end{aligned}
$$

Some data has been collected in stage 1 .

The two objective functions in (3.19) correspond to the two criteria specified in (3.13) and (3.16) in Section 3.2.1. These two criteria have been established through the mathematical derivations above as functions of the design $\eta_{d}$ and the prior information. The latter has been obtained from the first stage of the Bayesian procedure (Section 3.1) and includes $f(x, \widehat{\boldsymbol{\theta}}), \Pi(\boldsymbol{\theta})$, and $g(x, \widehat{\boldsymbol{\gamma}})$. Notice that we need to substitute the estimates $\widehat{\boldsymbol{\theta}}$ and $\widehat{\boldsymbol{\gamma}}$ for 
their true values in the formulas (3.7)- (3.18). The design $\eta_{d}$ represents the decision variables in (3.19) yet to be determined, and the optimum values of $\eta_{d}$ has been found through numeric algorithms.

However, solving (3.19) for the optimum design is a challenging task due to two special features of the problem. First, (3.19) has multiple objectives. As already mentioned, the existing experimental design approaches barely take into account multiple objectives, and optimizing the design with respect to a single objective often leads to unacceptable results with respect to other objectives. In the few design work where more than one design criterion are considered, two approaches have been used which basically convert the multi-objective optimization problem into a single-objective one. One approach is to form a weighted average of all the criteria [68-70]. The other approach is to move all but one objective to the constraint set. There are two major drawbacks with these methods: (i) In practice, it is usually very difficult to specify good weights or constraining values that reflect the researcher's preferences, even for someone familiar with the problem domain. Sometimes small perturbation in those parameters can lead to quite different solutions (designs). (ii) Both methods only return a single solution rather than a set of solutions, so they do not allow for the examination of various trade-offs including the ones incorporated (3.19) as well as those hard to be specified mathematically. To overcome these drawbacks, we propose to solve (3.19) using multi-objective metaheuristics [19,71, 72], and seek to find a number of solutions close to the Pareto optimal front, which is a set of non-dominated solutions where no objective can be improved without worsening at least one other objective. Specifically, the strength Pareto evolutionary algorithm developed by Thiele et al. [73] was adapted to approach the problem (3.19), and the resulting solutions provided a candidate pool of diverse designs that excel in terms of both criteria. These designs can be further evaluated based on their practicality and the one that achieves the best trade offs could be selected as the approximate optimum design. 
The second difficulty in solving (3.19) lies in computation of the criteria (3.13) and (3.16), which requires the evaluation of high dimensional integrals. The evaluation of the multivariate integral in mathematical models can rarely be calculated analytically. Number theoretic method and polynomial-based method are two primary numeric methods to approximate the multivariate integral. Monte Carlo method is the best representative of the first class, while second rule includes product rule, monomial rule, sparse grid, lattice rule and bayesian quadrature. In the former method, quasi-Monte Carlo methods are generally much more efficient than Monte Carlo and are able to handle very high-dimensional integrations [74]. The error of polynomial rule depends on higher order terms of a Taylor series expansion, which is much tighter than that of Monte Carlo methods [75] and numerical examples are given in [76], [77] and [78]. Monte Carlo, however, is the only method we can turn to in very high dimension [79].

Gaussian quadrature rule can get exact result for polynomials of degree $2 R-1$ or less with a suitable choice of $R$ nodes. That is, the more nodes used to integrate higher degree, the more accurate approximation integral is. In Gaussian quadrature, parameter spaces and weight function, such as $1, e^{-\theta^{2}}$, and $e^{-\theta}$, determine which orthogonal polynomial should be used. Gauss-Hermite qudarature is an extension of Gaussian quadrature method for approximating the value of integrals of the form

$$
\int_{-\infty}^{+\infty} e^{-\theta^{2}} f(\theta) d \theta \approx \sum_{i=1}^{R} w_{i} f\left(\theta_{i}\right)
$$

where $\theta_{i}$ is the roots of the hermite polynomial and $w_{i}$ is corresponding weights associate with it. Stround et al. [80] gives the table of nodes and weights for the Gauss-Hermite up to 136 points.

Cools [79] points out that in lower dimensions multiple Gaussian quadrature is the most important tool. Nodes of product rule is constructed by Kronecker product of onedimension nodes and weight is the multiplication of weights associated with one-dimension 
nodes. For product Gauss-Hermite qudarature rule, if the integrand contains multivariate normal distribution, Cholesky decomposition of the variance-covariance matrix can be used the change the variables. The general formula associated multivariate normal distribution with mean $\boldsymbol{\mu}$ and variance-covariance matrix $\Sigma$

$$
\int_{R^{k}} f(\boldsymbol{\theta}) \frac{1}{(2 \pi)^{k / 2}|\Sigma|^{1 / 2}} \exp \left\{-\frac{1}{2}(\boldsymbol{\theta}-\boldsymbol{\mu})^{T} \Sigma^{-1}(\boldsymbol{\theta}-\boldsymbol{\mu})\right\} d \boldsymbol{\theta}
$$

can be transformed by the setting $\mathbf{y}=\frac{\Sigma_{\Delta}^{-1}(\boldsymbol{\theta}-\boldsymbol{\mu})}{\sqrt{2}}$, where $\Sigma_{\Delta}$ is the cholesky factor of $\Sigma$, to

$$
\frac{1}{(\pi)^{k / 2}} \int_{R^{k}} f\left(\boldsymbol{\mu}+\Sigma_{\Delta} \sqrt{2} \mathbf{y}\right) \exp \left(-\mathbf{y}^{2}\right) d \mathbf{y}
$$

which can be integrated with product rule. Skrainka et al. [76] points out that there are two benefits of changing the variables: one is normal density disappears from the sum of the integration and the other is the variance covariance matrix is dropped out of the weights. Finally, product Gauss-Hermite rule would be used to integrate the two objectives in (3.19). 


\section{Chapter 4}

\section{Empirical Study}

In this section, the proposed two-stage design procedure is illustrated by a simulation study, which is based on sampling through computer experiments whose outputs mimic the

dose-response data from real experiments. Specifically, we evaluated the relative efficiency of the proposed design over the naive design of experiments. Given the same total sample size, would the optimum design lead to a more accurate and precise dose-response model than the traditional design? How much improvement can be achieved in the quality of the estimates? Simulation experiments are able to quantify these questions. Two nonlinear models are investigated: one is assumed to have increasing variance, and the other involves bell-shape variance function.

\subsection{Case 1: Nonlinear model with increasing variance}

In this case, assume underlying dose response curve is

$$
f(x, \boldsymbol{\theta})=\frac{\theta_{1}}{(1-x)^{\theta_{2}}}
$$

where $x$ is the dose level with design range $[0.1,0.95]$ and true parameter value $\boldsymbol{\theta}_{0}=(10,1)$. And assumed underlying variance model is

$$
g(x, \gamma)=\frac{\gamma_{1}}{(1-x)^{\gamma_{2}}}
$$


with true parameter value $\gamma_{0}=(25,0.5)$, which is similar to data in Figure 3.2.For $\mathrm{BMR}=5 \%$ (Section 3.2.1), true BMD is calculated as follows

$$
\begin{aligned}
\mathrm{BMD} & =f^{-1}\left(5 \% \frac{10}{(1-0)^{1}}+\frac{10}{(1-0)^{1}}\right) \\
& =0.0476
\end{aligned}
$$

\subsubsection{First-stage Experimentation and Modeling}

At this first stage of the Bayesian procedure (Figure 4.1a), some preliminary experiments were performed, and based on these initial data, prior information regarding the doseresponse curve and the data variability were obtained. The initial design consists of 3 points $(0.1,0.3082,0.95)$, equally spaced on logarithm scale on design range $[0.1,0.95]$, with 10 simulation replications at each point. Figure 4.1a provides the initial data set obtained, along with the fitted dose-response curve: $f(x, \hat{\boldsymbol{\theta}})=\frac{9.9732}{(1-x)^{1.0032}}$. Based on the data, we can then estimate variance model (4.2) using normal least square method and get variance model $g(x, \hat{\gamma})=\frac{15.29416}{(1-x)^{0.725356}}($ Figure 4.1b)

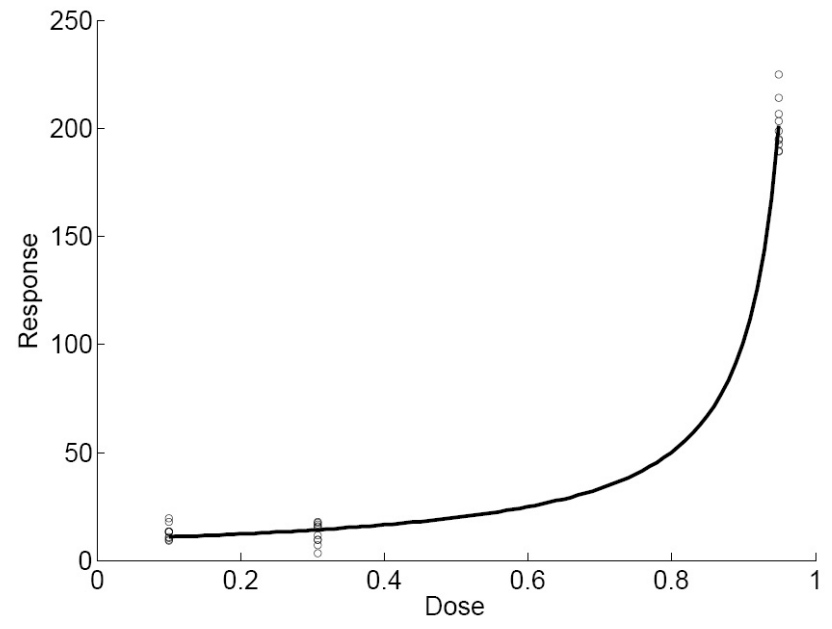

(a) Initial design and the fitted curve.

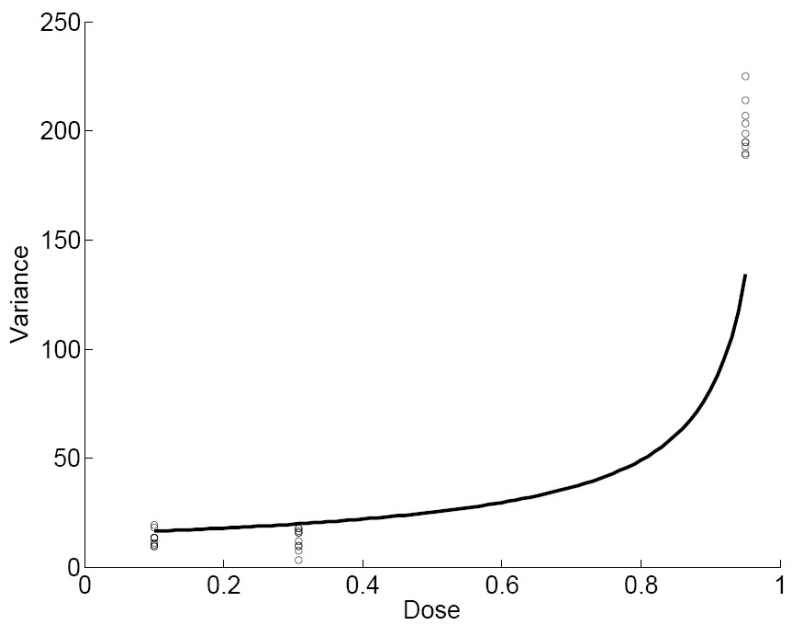

(b) Initial design and the fitted variance.

Figure 4.1: Initial design for case 1 
If we assume $\hat{\boldsymbol{\theta}}$ follows multivariate normal distribution, and use the wild bootstrap (Section 3.1.2) with $R=999$ to fit simulated data with dose-response curve model (4.1), we can get

$$
\hat{\boldsymbol{\theta}} \sim M N\left(\left(\begin{array}{l}
9.9732 \\
1.0032
\end{array}\right),\left(\begin{array}{cc}
0.7576 & -0.0257 \\
-0.0257 & 0.0009
\end{array}\right)\right),
$$

where $M N$ means multivariate normal distribution.

\subsubsection{Second-stage Design of Experiments}

Suppose we would like to augment $N=100$ samples to the initial design. Two objectives in (3.19) and constraints in (3.20) could be writhen as

$$
\begin{aligned}
\text { Objective 1: } & \max _{\eta_{d}} \int_{R^{2}}\left|\sum_{i=1}^{5} w_{i} \mathbf{I}\left(x_{i}, \boldsymbol{\theta}, \boldsymbol{\gamma}\right)\right| \frac{1}{\left.(2 \pi)|\Sigma|\right|^{1 / 2}} \exp \left\{-\frac{1}{2}(\boldsymbol{\theta}-\boldsymbol{\mu})^{T} \Sigma^{-1}(\boldsymbol{\theta}-\boldsymbol{\mu})\right\} d \boldsymbol{\theta} \\
\text { Objective 2: } \quad & \min _{\eta_{d}} \int_{R^{2}} \operatorname{tr}\left[\left(\frac{\partial f(0.0475, \boldsymbol{\theta})}{\partial \boldsymbol{\theta}}\right)\left(\frac{\partial f(0.0475, \boldsymbol{\theta})}{\partial \boldsymbol{\theta}}\right)^{T} \sum_{i=1}^{5} w_{i} \mathbf{I}\left(x_{i}, \boldsymbol{\theta}\right)^{-1}\right] \\
& \frac{1}{(2 \pi)|\Sigma|^{1 / 2}} \exp \left\{-\frac{1}{2}(\boldsymbol{\theta}-\boldsymbol{\mu})^{T} \Sigma^{-1}(\boldsymbol{\theta}-\boldsymbol{\mu})\right\} d \boldsymbol{\theta} \\
\text { With } \quad & \eta_{d}=\left\{\left(x_{i}, w_{i}\right) ; i=1,2, \ldots, 5\right\} \\
\text { Subject to: } \quad & x_{i} \in[0.1,0.95], \text { and } \sum_{i=1}^{5} w_{i}=1 \\
& \left(x_{3}, x_{4}, x_{5}\right)=(0.1,0.3082,0.95) \\
& w_{i} \geq \frac{10}{130} ; i=3,4,5
\end{aligned}
$$

Notice that since gamultiobj, controlled elitist genetic algorithm in Matlab, can only be used to find minima of multiple functions, the first objective function is needed to be converted into a minimization problem by multiplying the coefficients of the objective function by -1 . And the objective value of original maximum problem is just -1 times optimal objective value of the converted minimum problem. With gamultiobj in Matlab, we can get a set of optimal designs on Pareto front, graphically illustrated in Figure 4.2 and specifically detailed in Table A.1, subjected to these four constraints. Each row in Table A.1, corresponding to 
one star in Figure 4.2, represents a design, which is consisted of dose levels in columns 1-5 and corresponding weights in columns 6-10. For instance, row 9 in Table A.1 is design

$$
\left(\begin{array}{ccccc}
0.5726 & 0.7217 & 0.1 & 0.3082 & 0.95 \\
0.15273 & 0.16893 & 0.18413 & 0.18798 & 0.30690
\end{array}\right) \text {. }
$$

For this design we would allocate 20, 22, 24, 24, and 40 samples to dose level 0.5726, 0.7217, $0.1,0.3082$, and 0.95 respectively.

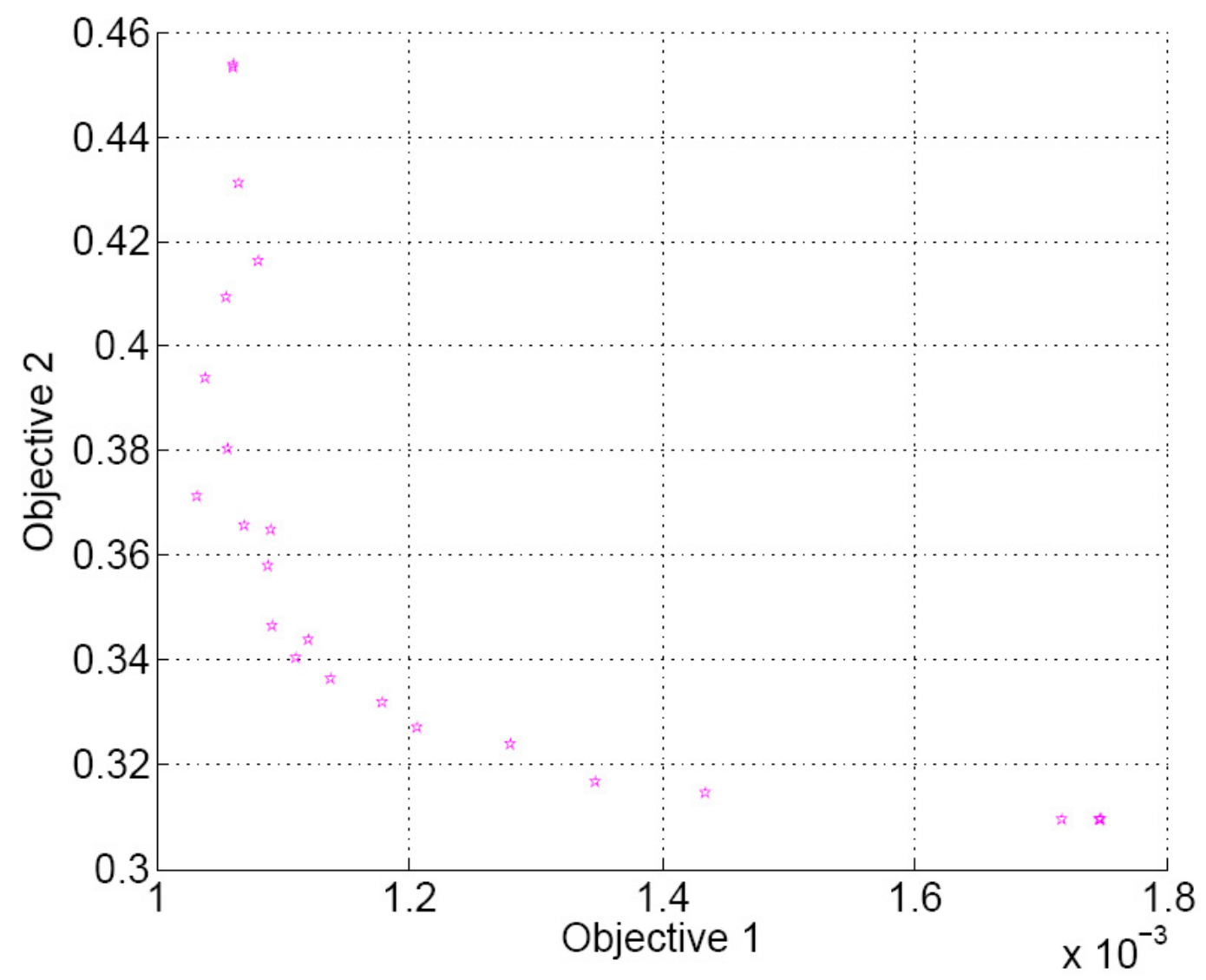

Figure 4.2: Optimal Pareto front of two-stage design procedure for case 1. The first objective is square root of first objective, while second objective is standard deviation of estimated variance 


\subsubsection{Design Comparison}

To demonstrate that the optimal design is more efficiency given the same amount of samples, we compare optimum designs on the Pareto front with traditional design in both generalized variance of parameter estimate and variance of estimated BMD. Traditional design (Table 4.1) is a design with five dose levels equally spaced on the logarithm scale of design region $[0.1,0.95]$ and equal weights on each dose levels in the second stage of design. We can see that design 9-17 of Table A.1, which is also in Table4.2, are better in both objectives than the traditional design; other designs, superior to tradition design in one objective, are worse in the other objective. For increasing pattern of variance, more weights on dose levels with large variance leads to smaller value of generalized variance. For example, design 1 of Table 4.1 with largest weight 0.48494 to dose level 0.95 , in which variance is largest, has smallest objective value compared with other designs on Pareto front. Since more designs done around estimated BMD can reduce variance of it, design 24 of Table A.1 allocate weight 0.76489 to dose level 0.6365 , which is closest to estimated BMD, to achieve smallest value in the second objective.

Table 4.1: Result of traditional design for case 1.

\begin{tabular}{|c|c|c|c|c|c|c|c|}
\hline & 1 & 2 & 3 & 4 & 5 & $|\operatorname{Var}(\hat{\boldsymbol{\theta}})|$ & $\operatorname{Stdev}(B \hat{M} D)$ \\
\cline { 1 - 7 } $\mathbf{x}$ & 0.1 & 0.1756 & 0.3082 & 0.5411 & 0.95 & \multirow{2}{*}{$1.55 \mathrm{E}-06$} & \multirow{2}{*}{$3.6738 \mathrm{E}-01$} \\
\hline weight & 0.2308 & 0.15385 & 0.2308 & 0.15385 & 0.2308 & & \\
\hline
\end{tabular}

\subsection{Case 2: Nonlinear model with bell shape variance}

Now, we consider another nonlinear model with bell-shape variance. In this case, assume underlying dose response curve is

$$
f(x, \boldsymbol{\theta})=\theta_{1} /\left(1+\exp \left(\left(\theta_{2}-x\right) / \theta_{3}\right)\right),
$$


Table 4.2: Part of optimal result of proposed two-stage design procedure for case 1.

\begin{tabular}{|c|c|c|c|c|c|c|c|c|c|c|c|c|}
\hline Number & $\mathrm{x} 1$ & $\mathrm{x} 2$ & $\mathrm{x} 3$ & $\mathrm{x} 4$ & $\mathrm{x} 5$ & $\mathrm{w} 1$ & $\mathrm{w} 2$ & $\mathrm{w} 3$ & $\mathrm{w} 4$ & w5 & $|\operatorname{Var}(\hat{\boldsymbol{\theta}})|$ & $\operatorname{Stdev}(B \hat{M} D)$ \\
\hline 9 & 0.5726 & 0.7217 & 0.1 & 0.3082 & 0.95 & 0.15273 & 0.16893 & 0.18413 & 0.18798 & 0.30690 & $1.14420 \mathrm{E}-06$ & $3.65630 \mathrm{E}-01$ \\
\hline 10 & 0.5238 & 0.7353 & 0.1 & 0.3082 & 0.95 & 0.16041 & 0.15212 & 0.17700 & 0.21722 & 0.29383 & $1.18988 \mathrm{E}-06$ & $3.64887 \mathrm{E}-01$ \\
\hline 11 & 0.5790 & 0.7192 & 0.1 & 0.3082 & 0.95 & 0.21409 & 0.15230 & 0.17354 & 0.17692 & 0.28386 & $1.18479 \mathrm{E}-06$ & $3.57975 \mathrm{E}-01$ \\
\hline 12 & 0.6228 & 0.6912 & 0.1 & 0.3082 & 0.95 & 0.31747 & 0.15072 & 0.16602 & 0.10350 & 0.26321 & $1.19346 \mathrm{E}-06$ & $3.46499 \mathrm{E}-01$ \\
\hline 13 & 0.6876 & 0.8216 & 0.1 & 0.3082 & 0.95 & 0.36494 & 0.05113 & 0.16166 & 0.18086 & 0.24204 & $1.25637 \mathrm{E}-06$ & $3.43770 \mathrm{E}-01$ \\
\hline 14 & 0.7546 & 0.8391 & 0.1 & 0.3082 & 0.95 & 0.43053 & 0.03063 & 0.16266 & 0.14151 & 0.23543 & $1.23360 \mathrm{E}-06$ & $3.40309 \mathrm{E}-01$ \\
\hline 15 & 0.7147 & 0.8606 & 0.1 & 0.3082 & 0.95 & 0.45130 & 0.02863 & 0.15023 & 0.14957 & 0.22101 & $1.29601 \mathrm{E}-06$ & $3.36448 \mathrm{E}-01$ \\
\hline 16 & 0.7060 & 0.8734 & 0.1 & 0.3082 & 0.95 & 0.48670 & 0.02717 & 0.13558 & 0.15187 & 0.19853 & $1.39113 \mathrm{E}-06$ & $3.31681 \mathrm{E}-01$ \\
\hline 17 & 0.6894 & 0.7921 & 0.1 & 0.3082 & 0.95 & 0.50594 & 0.03715 & 0.13097 & 0.13924 & 0.18695 & $1.45517 \mathrm{E}-06$ & $3.27070 \mathrm{E}-01$ \\
\hline
\end{tabular}

where $x$ is the dose level in design range $[1.5,4]$ and true parameter value $\boldsymbol{\theta}_{0}=(50,2.5,0.3)$, which is similar to data in Figure2.1. And assumed underlying variance model is

$$
g(x, \gamma)=x^{\gamma_{1}}(4.5-x)^{\gamma_{2}}
$$

with true parameter value $\gamma_{0}=(1.5,4)$. For $\mathrm{BMR}=5 \%$, true BMD is calculated as follows

$$
\begin{aligned}
\mathrm{BMD} & =f^{-1}\left(5 \% \frac{50}{(1+\exp ((2.5-x) / 0.3))}+\frac{50}{(1+\exp ((2.5-x) / 0.3))}\right) \\
& =0.0146
\end{aligned}
$$

\subsubsection{First-stage Experimentation and Modeling}

At this first stage of the Bayesian procedure (Figure 4.3a), some preliminary experiments were performed, and based on these initial data, prior information regarding the doseresponse curve and the data variability were obtained. Initial design, consisted of 3 points $(1.5,2.449,4)$ with 10 simulation replications at each point, is equally spaced on the natural logarithm on design region $[1.5,4]$, along with the fitted dose-response curve: $f(x, \hat{\boldsymbol{\theta}})=49.7645 /(1+\exp ((2.4947-x) / 0.3431))$. We can then estimate variance model (4.5) using normal least square method and get variance model $g(x, \hat{\gamma})=x^{2.1549}(4.5-x)^{0.6061}$ (Figure 4.3b). 


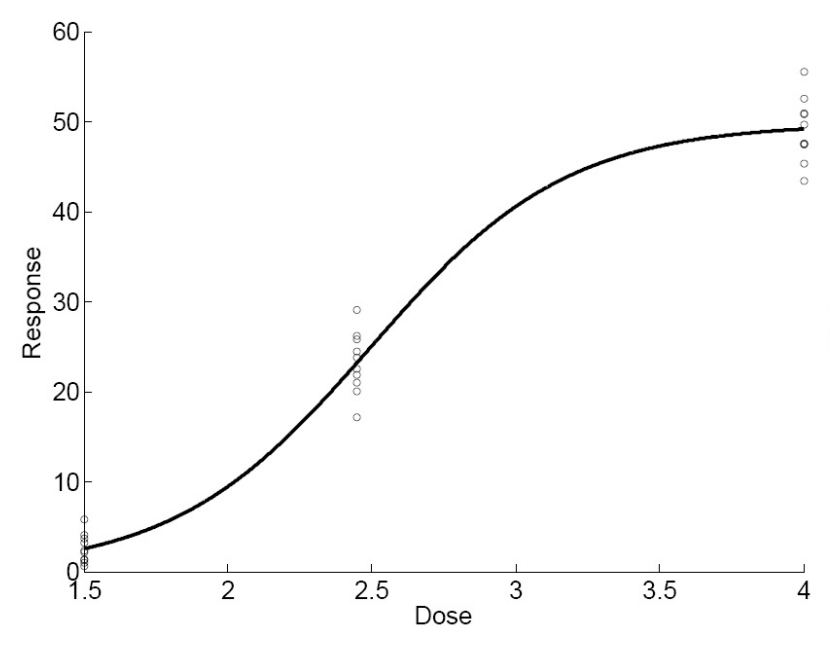

(a) Initial design and the fitted curve.

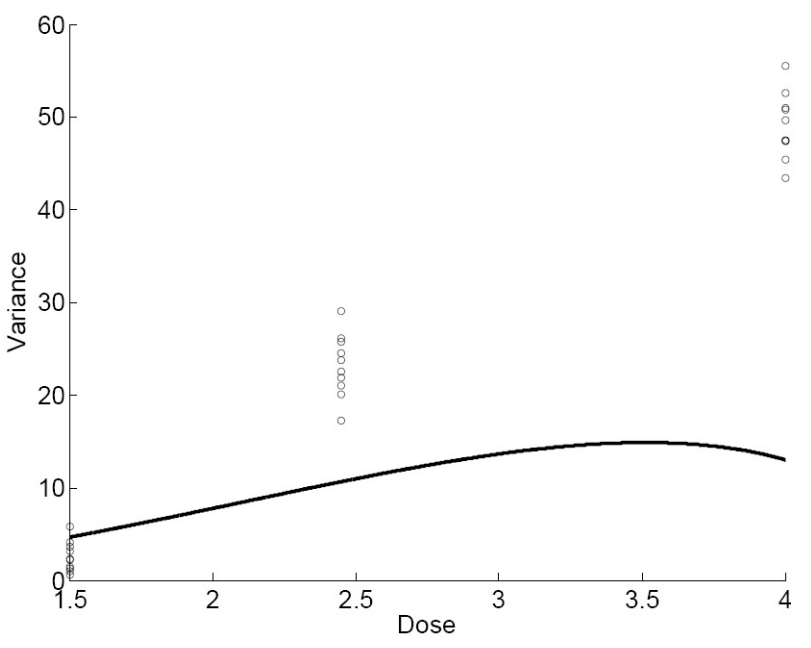

(b) Initial design and the fitted variance.

Figure 4.3: Initial design for case 2.

If we assume $\hat{\boldsymbol{\theta}}$ follows multivariate normal distribution, and use the wild bootstrap with $R=999$ to fit simulated data with dose-response curve model (4.4), we can get

$$
\hat{\boldsymbol{\theta}} \sim M N\left(\left(\begin{array}{l}
49.7645 \\
2.4947 \\
0.3431
\end{array}\right),\left(\begin{array}{ccc}
1.557 & 0.0273 & 0.0115 \\
0.0273 & 0.0014 & 0.0006 \\
0.0115 & 0.0006 & 0.0009
\end{array}\right)\right)
$$

where $M N$ means multivariate normal distribution. 


\subsubsection{Second-stage Design of Experiments}

Similarly, we set the number of augmented designs in second-stage design to be 100. Objectives(3.19) and constraints in (3.20) could be writhen as

Objective 1: $\max _{\eta_{d}} \int_{R^{3}}\left|\sum_{i=1}^{5} w_{i} \mathbf{I}\left(x_{i}, \boldsymbol{\theta}, \boldsymbol{\gamma}\right)\right| \frac{1}{(2 \pi)^{3 / 2}|\Sigma|^{1 / 2}} \exp \left\{-\frac{1}{2}(\boldsymbol{\theta}-\boldsymbol{\mu})^{T} \Sigma^{-1}(\boldsymbol{\theta}-\boldsymbol{\mu})\right\} d \boldsymbol{\theta}$

Objective 2: $\min _{\eta_{d}} \int_{R^{3}} \operatorname{tr}\left[\left(\frac{\partial f(0.0167, \boldsymbol{\theta})}{\partial \boldsymbol{\theta}}\right)\left(\frac{\partial f(0.0167, \boldsymbol{\theta})}{\partial \boldsymbol{\theta}}\right)^{T} \sum_{i=1}^{5} w_{i} \mathbf{I}\left(x_{i}, \boldsymbol{\theta}\right)^{-1}\right]$

$$
\frac{1}{(2 \pi)^{3 / 2}|\Sigma|^{1 / 2}} \exp \left\{-\frac{1}{2}(\boldsymbol{\theta}-\boldsymbol{\mu})^{T} \Sigma^{-1}(\boldsymbol{\theta}-\boldsymbol{\mu})\right\} d \boldsymbol{\theta}
$$

With $\quad \eta_{d}=\left\{\left(x_{i}, w_{i}\right) ; i=1,2, \ldots, 5\right\}$

Subject to: $\quad x_{i} \in[1.5,4]$, and $\sum_{i=1}^{5} w_{i}=1$

$$
\left(x_{3}, x_{4}, x_{5}\right)=(1.5,2.449,4)
$$

$$
w_{i} \geq \frac{10}{130} ; i=3,4,5
$$

Finally, we can get graphically Pareto front (Figure 4.4) and detailed result (Table 4.3) subjected to constraints on weights and dose levels with gamultiobj function in Matlab.

\subsubsection{Design Comparison}

We can see that design 1-16 of optimum design of proposed method (Table A.2), which is also Table 4.4, are better in both objectives than the tradition design (Table 4.3); design 17-24 of Table A.2 are better than tradition design only in the second objective. Since fitted variance is first increase from 1.5-3.515 and then decrease to 4 (Figure 4.3b), which is almost increasing the whole design region, more weights are needed on begin and especially end of the design space to achieve better the objective value of first objective. For instance, design 1 of Table A.2 with weight 0.361272 near the end of the design region has the smaller value of first objective than any other design. Better objective value of second objective can be achieved by putting more weights near the BMD because of almost increasing variance pattern of whole design region of the design. For example, last design of Table A.2 with 


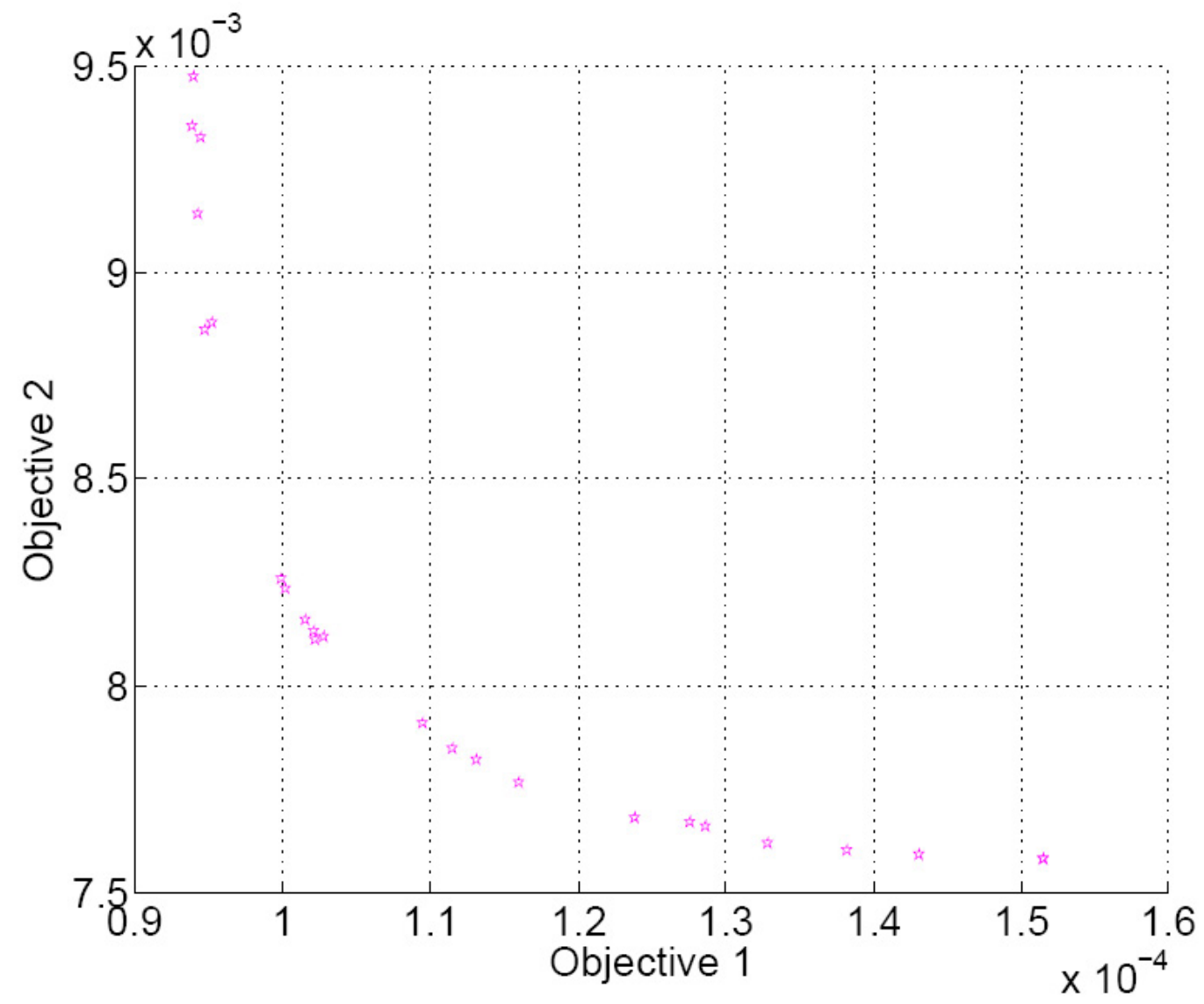

Figure 4.4: Optimal Pareto front of two-stage design procedure for case 2. The first objective is square root of first objective, while second objective is standard deviation of estimated variance

weight 0.557827 on dose level 1.737388 , which is closer to estimate BMD than other design on Pareto front.

Table 4.3: Result of traditional design for case 2 .

\begin{tabular}{|c|c|c|c|c|c|c|c|}
\hline & 1 & 2 & 3 & 4 & 5 & $|\operatorname{Var}(\hat{\boldsymbol{\theta}})|$ & $\operatorname{Stdev}(B \hat{M} D)$ \\
\hline $\mathbf{x}$ & 1.5 & 1.9168 & 2.449 & 3.1302 & 4 & \multirow{2}{*}{$1.414 \mathrm{E}-08$} & \multirow{2}{*}{$9.9839 \mathrm{E}-03$} \\
\cline { 1 - 6 } weight & 0.2308 & 0.1538 & 0.2308 & 0.1538 & 0.2308 & & \\
\hline
\end{tabular}




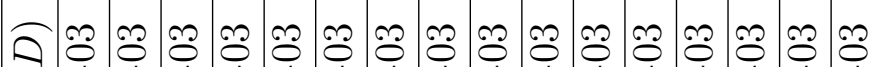

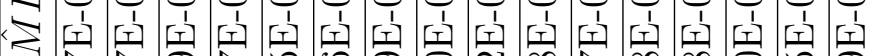

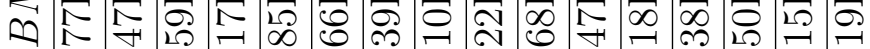

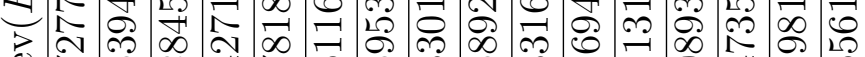

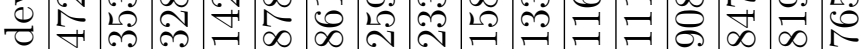

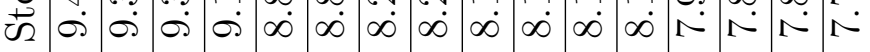

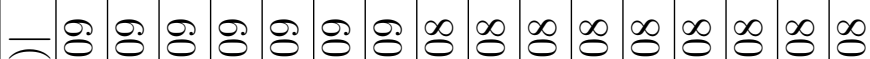
(2) 1 (1)

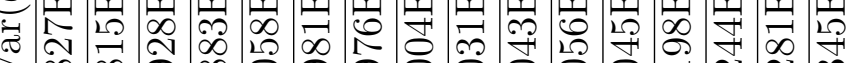
న

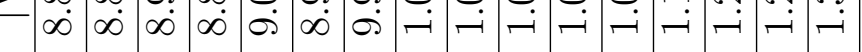

N

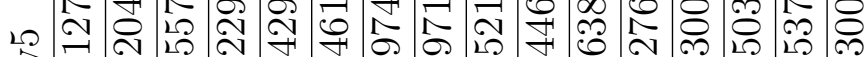

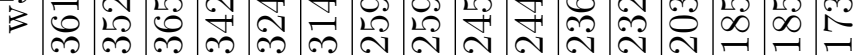
ம் $\mathscr{1}$

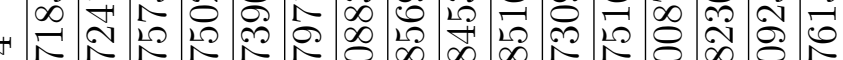

BN N N N N N

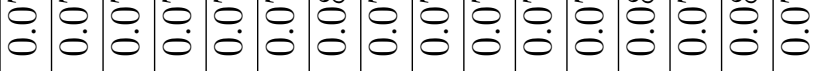
๓ m

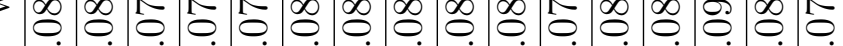
$\begin{array}{lllllllllllllllll}0 & 0 & 0 & 0 & 0 & 0 & 0 & 0 & 0 & 0 & 0 & 0 & 0 & 0 & 0 & 0\end{array}$

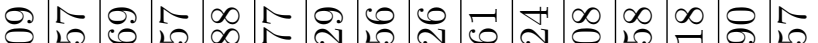
$\checkmark$ m

B N $\underset{\sim}{\sim}$ 只 $\begin{array}{llllllllllllllll}0 & 0 & 0 & 0 & 0 & 0 & 0 & 0 & 0 & 0 & 0 & 0 & 0 & 0 & 0 & 0\end{array}$ ケ

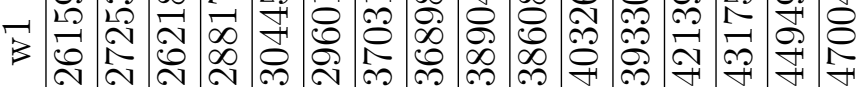

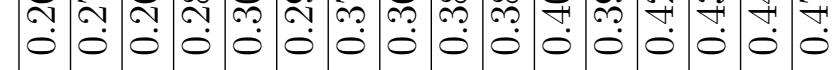

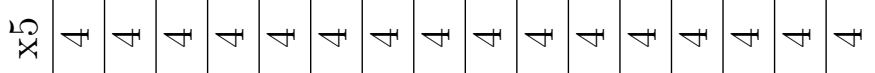

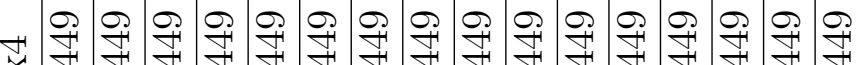

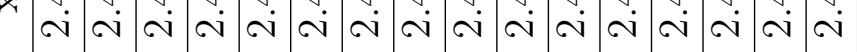
\%

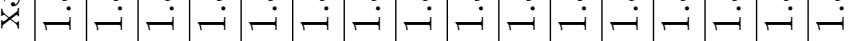
S)

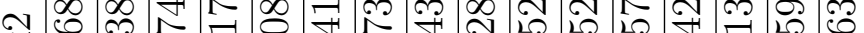
我

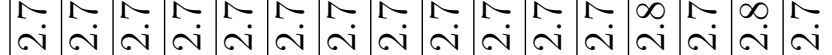

舟

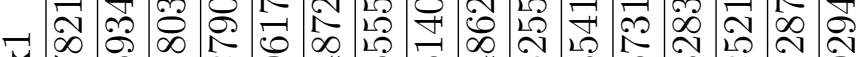

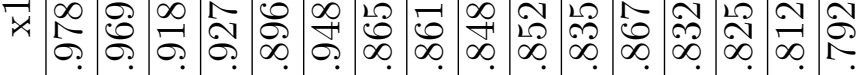

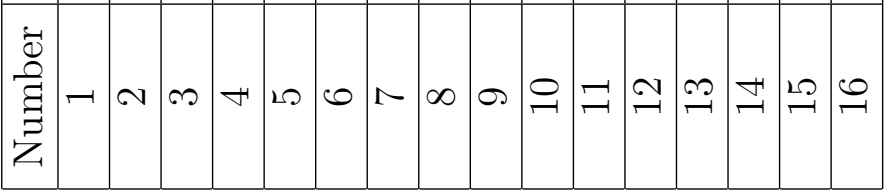




\section{Chapter 5}

\section{Conclusions and future study}

One of the most fundamental steps in assessing the risk of a nanomaterial is to understand and properly characterize its dose-response relationship based on biological experiments. Because of costs, ethics, or other limitations on resources or time, sample sizes

are usually restricted and efficient use of available resources is critical. Thus, the design of experiments, i.e., the selection of experimental doses and the allocation of animals, plays an important role in the success of dose-response studies.

Efficient design for dose-response modeling is particularly challenging due to the special features of toxicity data. The objective of this work was to develop an experimental design procedure, which accommodates the nonlinear nature of dose-response curves and variance heterogeneity of toxicity data, to guide the dose selection and animal allocation in biological experiments for the efficient generation of dose-response relationships. The proposed design procedure is built in a two-stage Bayesian paradigm, which provides a statistically valid mechanism to utilize prior information for the design of future experiments. Most suitable dose-response as well as variance models are identified to describe the toxicity data. Bootstrapping, a computationally intensive resampling method as opposed to conventional statistical inference methods, are used to derive important information from the preliminary data required by the subsequent experimental design. To achieve practically useful designs, multiple design criteria are considered simultaneously, and multi-objective metaheuristics are adapted to search for a set of Pareto optimum designs, which allow for the evaluation of various trade-offs in practical experimental settings. The experimental 
design procedure has been evaluated through two simulation cases designed to mimic real toxicity data. It has been shown that the design resulting from our procedure is superior to the traditional "naive" design.

In the current procedure, the design criteria for the second-stage experiments is derived based on normality assumption of the experimental data. An immediate next step is to relax the normality assumption and develop a method to evaluate the design criteria for general data. The design optimization in the second stage will then be based on the design criteria that are appropriate for both normal and non-normal data.

Although recognized important in the statistical literature, such sophisticated experimental design methods appear not to have been used much in practice. We believe that this is at least partly due to the lack of an easy-to-use software that biologists feel comfortable with. To make it possible for biologists to directly design their experiments, we plan to develop a menu-driven VBA macro for Excel that implements the proposed two-stage design procedure.

The VBA macro tool provides the user with specific guidance as to what data to collect and what analysis to be performed. The tool depends on the user to obtain and input the data into excel spreadsheets, and to click several buttons to complete the entire process. Specifically, the steps that the user needs to take are described as follows. (i)The user opens an Excel spreadsheet containing the VBA macros, and selects the experimental design menu that is added to the standard Excel menus. (ii) The software prompts the user to input the preliminary data in the designated area of the spreadsheet, and once the data is in place, the user will be prompted to press a button to obtain the optimum designs for the next-stage experiments. The near Pareto optimum design set, along with their corresponding objective/criterion values, will then be displayed on the spreadsheet. (iii) The user evaluates each design in the solution set based on the two design criteria and her practical concerns, and select one for her experiments. After new experiments have been performed and the data 
put in the spreadsheet, the user can generate the dose-response curve and BMD estimates by clicking another button.

Note that Step (ii) executes the proposed design procedure described in Sections 3.1 and 3.2. Step (iii) provides the modeling and analysis once the data is complete. 


\section{Appendix A}

\section{Result of Empirical Study}

Table A.1 and A.2 is result of proposed two-stage design procedure for case 1 and case 2 respectively. 


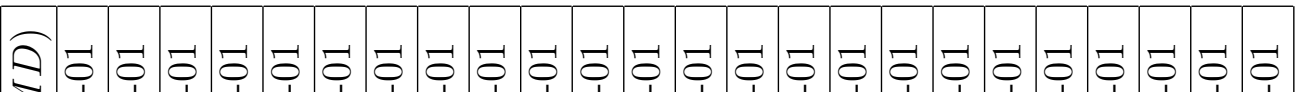

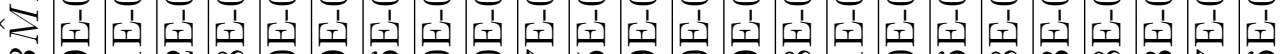

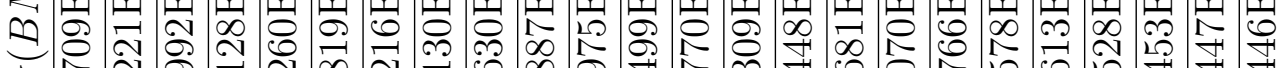

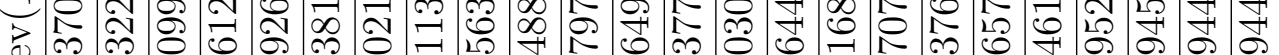

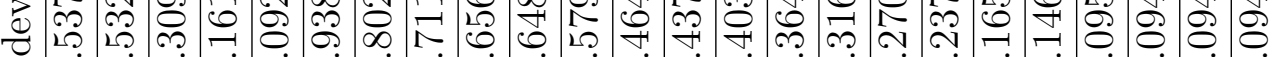

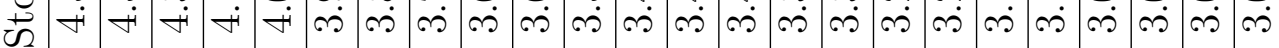

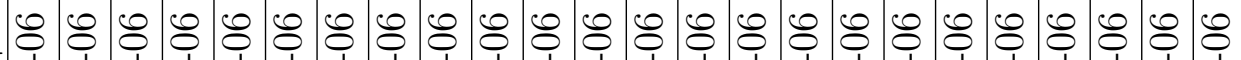

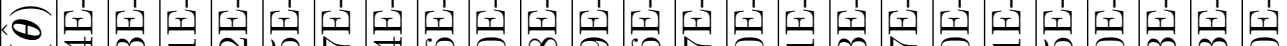

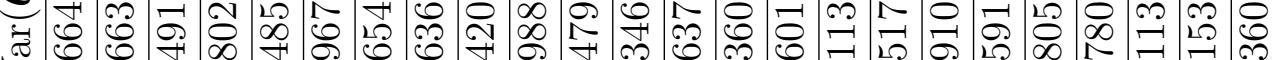

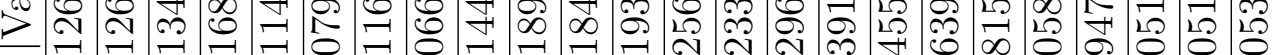

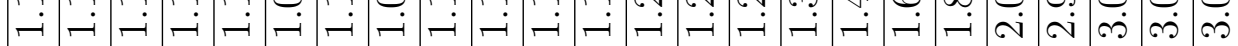

ஆ 上

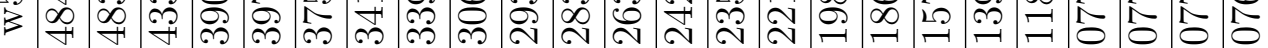

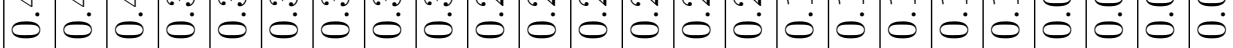
我

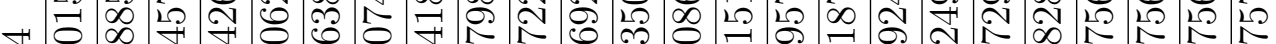

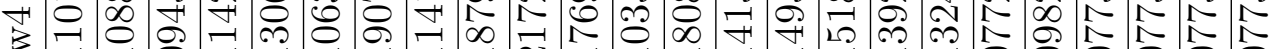
元

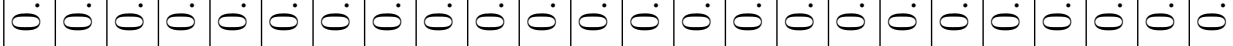
म

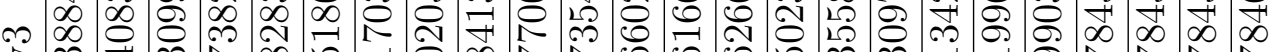

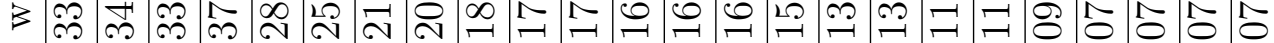

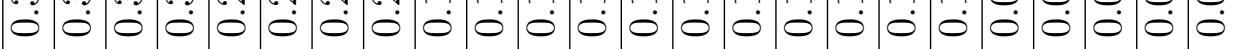
No N B

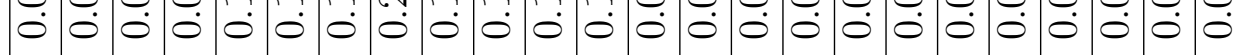
身

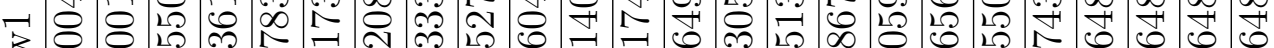
B ம

L メ

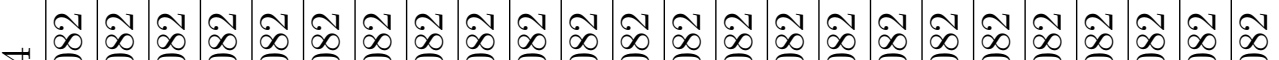

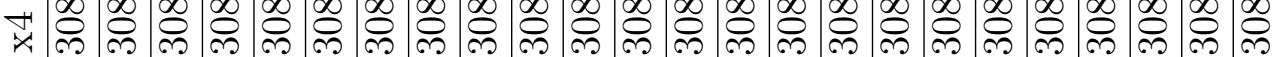

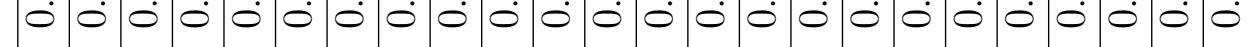

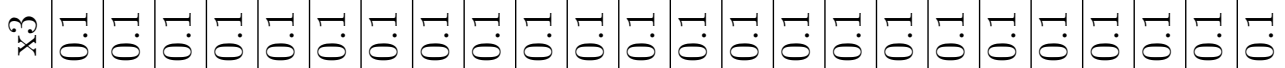
ヘִ ㄴ 幽弪

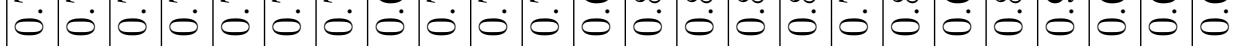

•

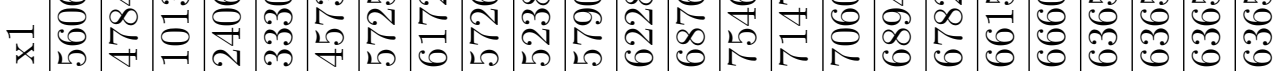

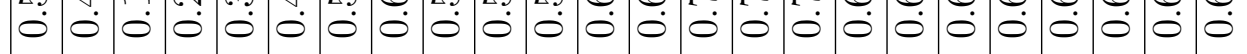

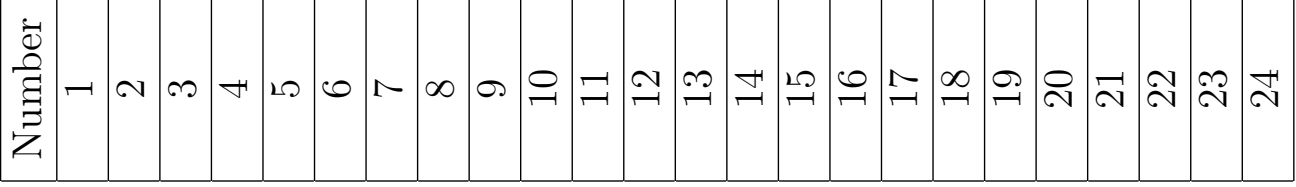


Оิ

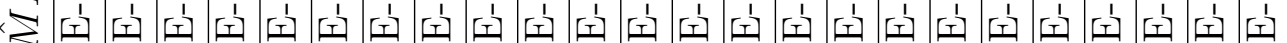

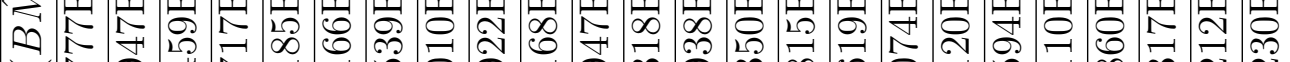

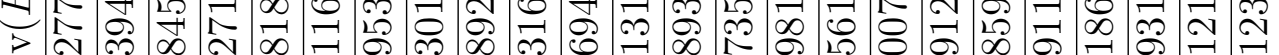

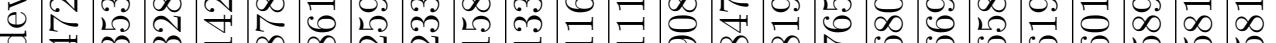

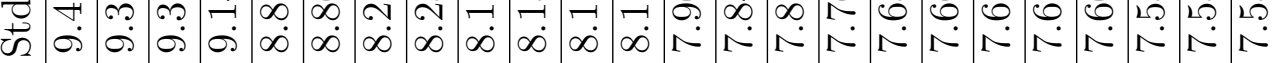

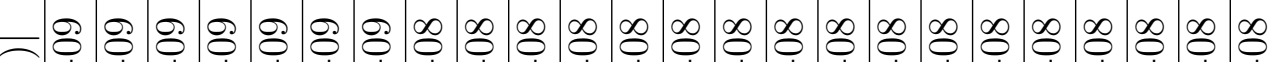

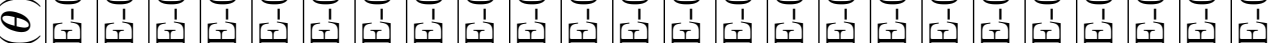

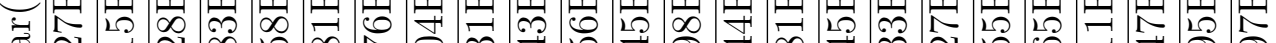

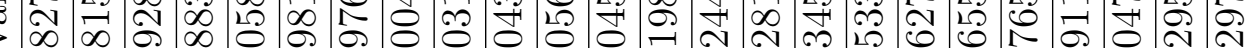

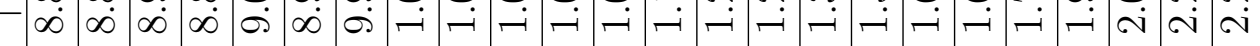
กิ

L

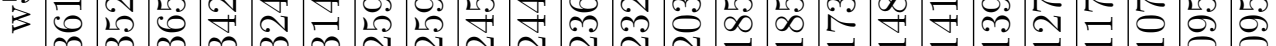
๑ே n

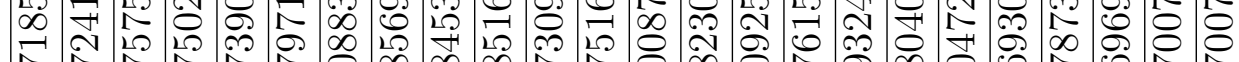
H 3 N $\left.\begin{array}{c}1 \\ 0\end{array}\right)$

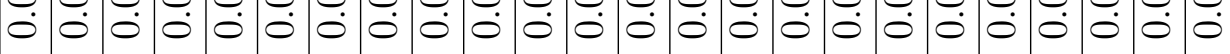

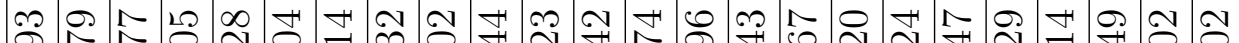
m 2 至

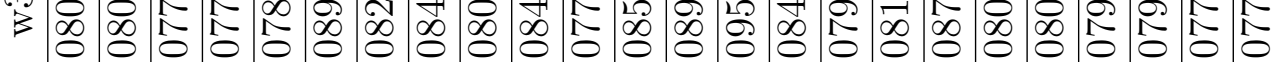

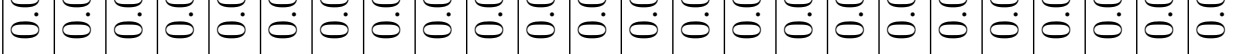

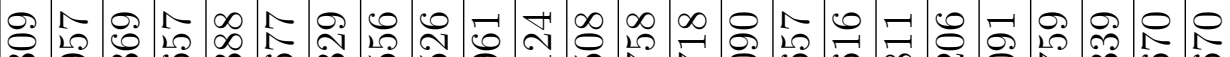
$\sim$ m

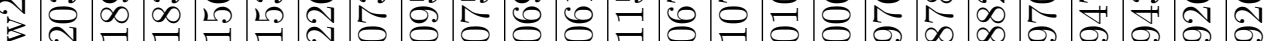

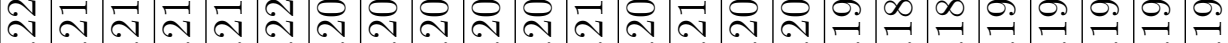

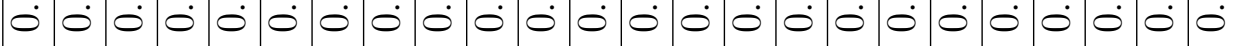
\%

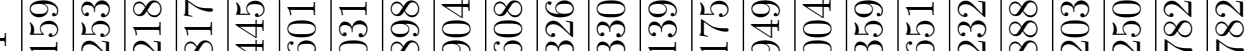
उ

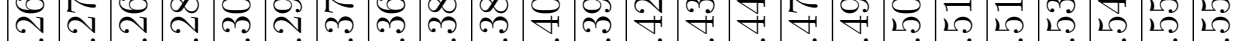

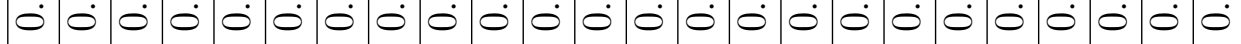

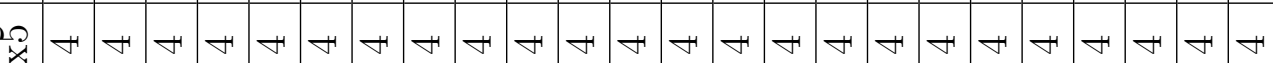

(2)

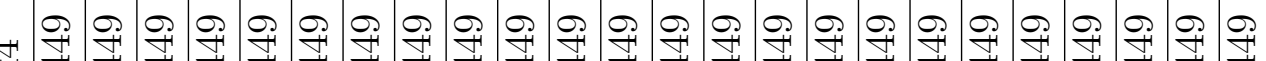

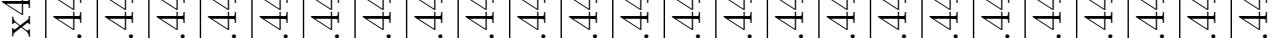

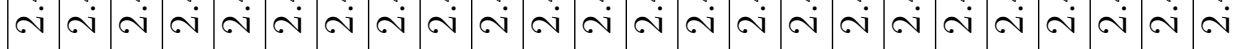
$\Re$ 藏 $\ddot{\sim}$

$\dot{4}$

$\frac{0}{\frac{\pi}{6}}$

2

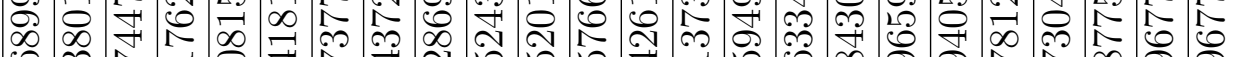

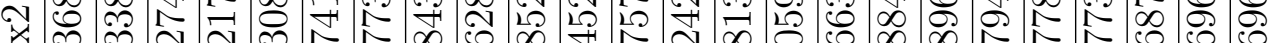

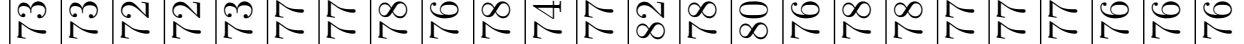

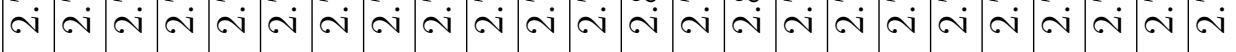

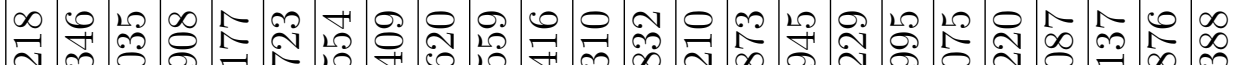

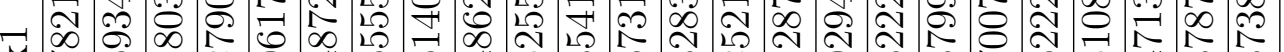

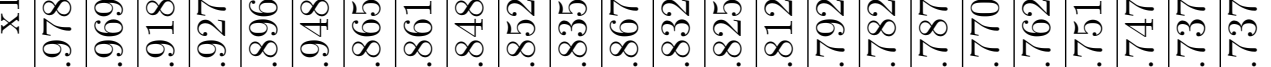

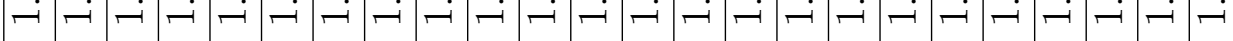

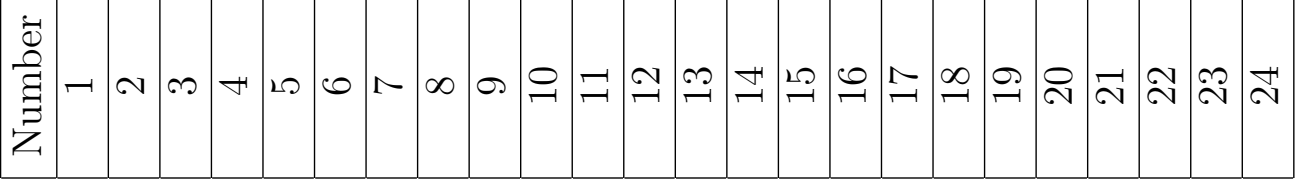




\section{References}

[1] National Academy of Sciences. Review of federal strategy for nanotechnology-related environmental, health, and safety research, 2009.

[2] L. Edler, K. Poirier, M. Dourson, J. Kleiner, B. Mileson, H. Nordmann, A. Renwickf, W. Slobg, K. Waltonf, and G. Wurtzenh. Mathematical modelling and quantitative methods. Food and Chemical Toxicology, 40:283-326, 2002.

[3] F. Bretz, J. Hsu, J. Pinheiro, and Y. Liu. Dose finding - a challenge in statistics. Biometrical Journal, 50:480-504, 2008.

[4] R.F. Hamilton, N.Q. Wu, D. Porter, M. Buford, M. Wolfarth, and A. Holian. Particle length-dependent titanium dioxide nanomaterials toxicity and bioactivity. Particle and Fibre Toxicology, 6:doi:10.1186/1743-8977-6-35, 2009.

[5] D.W. Porter, A.F. Hubbs, R.R. Mercer, N.Q. Wu, M.G. Wolfarth, K. Sriram, S. Leonard, L. Battelli, S. Schwegler-Berry, D.and Friend, M. Andrew, B.T. Chen, S. Tsuruoka, M. Endo, and V. Castranova. Mouse pulmonary dose- and time courseresponses induced by exposure to multi-walled carbon nanotubes. Toxicology, 269:136 $-147,2010$.

[6] W. Slob. Dose-response modeling of continuous endpoints. Toxicological Sciences, 66:298-312, 2002.

[7] S. Sand, D. von Rosen, P. Eriksson, A. Fredriksson, H. Viberg, K. Victorin, and A.F. Filipsson. Dose-response modeling and benchmark calculations from spontaneous behavior data on mice neonatally exposed to 2,2',4,4',5-pentabromodiphenyl ether. Toxicological Sciences, 81:491-501, 2004.

[8] G. Oberdorster, E. Oberdorster, and J. Oberdorster. Nanotoxicology: An emerging discipline evolving from studies of ultrafine particles. Environmental Health Perspectives, 113:823-839, 2005.

[9] A.C. Atkinson and A.N. Donev. Optimum Experimental Designs. Clarendon Press, 1992. 
[10] G. A. F. Seber and C. J. Wild. Nonlinear regression. 2003.

[11] G. Dunn. Optimal designs for drug, neurotransmitter and hormone receptor assays. Statistics in Medicine, 7:805-815, 1988.

[12] H. Dette and W.K. Wong. Optimal designs when the variance is a function of the mean. Biometrics, 55:925-929, 1999.

[13] V.V. Fedorov, R.C. Gagnon, and S.L. Leonov. Design of experiments with unknown parameters in variance. Applied Stochastic Models in Business and Industry, 18:207-218, 2002.

[14] B. Bogacka and F. Wright. Non-linear design problem in a chemical kinetic model with non-constant error variance. Journal of Statistical Planning and Inference, 128:633-648, 2005.

[15] F. Yang, B.E. Ankenman, and B.L. Nelson. Efficient generation of cycle time-throughput curves through simulation and metamodeling. Naval Research Logistics, 54:78-93, 2007.

[16] F. Yang, B.E. Ankenman, and B.L. Nelson. Cycle time percentile curves for manufacturing systems. volume 20, pages 628-643, 2008.

[17] F. Yang. Neural network metamodeling for cycle-time based performance profiles in manufacturing. European Journal of Operations Research, 205:172-185, 2010.

[18] K. Chaloner and I. Verdinelli. Bayesian experimental design: a review. Statistical Science, 10:273-304, 1995.

[19] K. Deb. Multi-Objective Optimization using Evolutionary Algorithms. John Wiley \& Sons, Chichester, 2001.

[20] B.E. Rollin. The regulation of animal research and the emergence of animal ethics: A conceptual history. Theoretical Medicine and Bioethics, 27:285-304, 2006.

[21] S. Festing and R. Wilkinson. The ethics of animal research. talking point on the use of animals in scientific research. The European Molecular Biology Organization Report, 8:526-530, 2007.

[22] L.A. Mitchell, J. Gao, R.V. Wal, A. Gigliotti, S.W. Burchiel, and J.D. McDonald. Pulmonary and systemic immune response to inhaled multiwalled carbon nanotubes. Toxicological Sciences, 100:203-214, 2007. 
[23] Y. Hochberg and A. C. Tamhane. Multiple Comparisons Procedures. Wiley, New York, 1987.

[24] J. C. Hsu. Multiple comparisons. 1996.

[25] K.S. Crump. Critical issues in benchmark calculations from continuous data. Critical Reviews in Toxicology, 32:133-153, 2002.

[26] A.F. Filipsson, S. Sand, J. Nilsson, and K. Victorin. The benchmark dose method-review of available models and recommendations for application in health risk assessment. Critical Reviews in Toxicology, 33:505-542, 2003.

[27] K.Z. Travis, I. Pate, and Z.K. Welsh. The role of benchmark dose in a regulatory context. Regulatory Toxicology and Pharmacology, 43:280-291, 2005.

[28] S. Sand, K. Victorin, and A. F. Filipsson. The current state of knowledge on the use of the benchmark dose concept in risk assessment. Journal of Applied Toxicology, 28:405-421, 2008.

[29] S. Leonov and S. Miller. An adaptive optimal design for the emax model and its application in clinical trials. Journal of Biopharmaceutical Statistics, 19:360-385(26), 2009.

[30] R. J. Kavlock, J. E. Schmid, and R. W. Setzer. A simulation study of the influence of study design on the estimation of benchmark doses for developmental toxicity. Risk Analysis, 16:399-410, 1996.

[31] D. Krewski, R. Smythe, and K. Y. Fung. Optimal designs for estimating the effective dose in developmental toxicity experiments. Risk Analysis, 22:1195-1205, 2002.

[32] K. Kuljus, D. V. Rosen, S. Sand, and K. Victorin. Comparing experimental designs for benchmark dose calculations for continuous endpoints. Risk Analysis, 26:1031-1043, 2006.

[33] H. Dette, A. Pepelyshev, and W. K. Wong. Optimal designs for dose-finding experiments in toxicity studies. Bernoulli, 15:124-145, 2009.

[34] Design of experiments for estimating parameters with unknown heterogeneity of the error variance, 1983. 
[35] A. DasGupta, S. Mukhopadhyay, and W.J. Studden. Compromise designs in heteroscedastic linear models. Journal of Statistical Planning and Inference, 32:363-384, 1992.

[36] W.K. Wong. A unified approach to the construction of mini-max designs. Biometrika, 79:611-619, 1992.

[37] W.K. Wong. Multifactor g-optimal designs with heteroscedastic errors. Journal of Statistical Planning and Inference, 40:127-133, 1994.

[38] A.C. Atkinson and R.D. Cook. D-optimum designs for heteroscedastic linear models. Journal of the American Statistical Association, 90:204-212, 1995.

[39] H. Dette and W.K Wong. Bayesian d-optimal designs on a fixed number of design points for heteroscedastic polynomial models. Biometrika, 85:869-882, 1998.

[40] H. Dette and A. Munk. Testing heterosedasticity in nonparamteric regression. Journal of the Royal Statistical Society Series B, 60:693-708, 1998.

[41] D.P. Wiens. Minimax robust designs and weights for approximately specified regression models with heteroscedastic errors. Journal of the American Statistician Association, 93:1440-1450, 1998.

[42] L.D. Brown and W.K. Wong. An algorithmic construction of optimal minimax designs for heteroscedastic linear models. Journal of Statistical Planning and Inference, 85:103$114,2000$.

[43] L. Tack, P. Goos, and M. Vandebroek. Efficient bayesian designs under heteroscedasticity. Journal of Statistical Planning and Inference, 104:469-483, 2002.

[44] H. Dette, F. Bretz, A. Pepelyshev, and J. Pinheiro. Optimal designs for dose-finding studies. Journal of the American Statistical Association, 103:1225-1237, 2008.

[45] P. H. Garthwaite and J. M. Dickey. Quantifying expert opinion in linear regression problems. Journal of the Royal Statistical Society. Series B (Methodological), 50(3):pp. 462-474, 1988.

[46] J. B. Kadane. Bayesian Methods and Ethics in a Clinical Trial Design. John Wiley \& Sons, New York, 1996.

[47] M.A. Clyde. Experimental design: A bayesian perspective. International Encyclopedia of the Social and Behavioral Sciences, 8:5075-5081, 2001. 
[48] H. Dette, D. Song, and W.K. Wong. Robustness properties of minimallysupported bayesian d-optimal designs for heteroscedastic models. The Canadian Journal of Statistics, 29:633-647, 2001.

[49] G.B.P. Box and W. J. Hill. Correcting inhomogeneity of variance with power transformation weight-ing. Technometrics, 16:385-389, 1974.

[50] R.D. Cook and S. Weisberg. Diagnostics for heteroscedasticity in regression. Biometrika, 70:1-10, 1983.

[51] R.J. Carroll and D. Ruppert. Robust estimation in heteroscedastic linear models. The Annals of Statistics, 10:429-441, 1982.

[52] E.L. Lehmann. Elements of Large-Sample Theory, New York, 2004.

[53] B. Efron and R. J. Tibshirani. An Introduction to the Bootstrap. Chapman \& Hall, 1994.

[54] A.C. Davison and D.V. Hinkley. Bootstrap Methods and Their Application. Cambridge University Press, Cambridge, 1997.

[55] W.J. Slob and M. Pieters. A probabilistic approach for deriving acceptable human intake limits and human health risks from toxicological studies: General framework. Risk Analysis, 18:787-798, 1998.

[56] R.Y. Rubinstein and D.P. Kroese. Simulation and the Monte Carlo Method, 2nd edition. John Wiley \& Sons, New York, 2007.

[57] B. Efron and G. Gong. A leisurely look at the bootstrap, the jackknife, and crossvalidation. The American Statistician, 37(1), 1983.

[58] D. A. Freedman. Bootstrapping regression models. Ann Statist, 9:1218-1228, 1981.

[59] E. Mammen. Bootstrap and wild bootstrap for high dimensional linear models. The Annals of Statistics, 21:255-285, 1993.

[60] E. Flachaire. A better way to bootstrap pairs. Economics Letters, 64:257-262, 1993.

[61] R. Y. Liu. Bootstrap procedure under some non-iid models. The Annals of Statistics, 16:1696-1708, 1988.

[62] C.F.J. Wu. Jackknife bootstrap and other resampling methods in regression analysis. The Annals of Statistics, 14:1261-1295, 1986. 
[63] J. G. MacKinnon. Bootstrap inference in econometrics. The Canadian Journal of Economics / Revue canadienne d'Economique, 35:615-645, 2002.

[64] D. Brownstone and R. Valletta. The bootstrap and multiple imputations: Harnessing increased computing power for improved statistical tests. The Journal of Economic Perspectives, 15:129-141, 2001.

[65] J. L. Horowitz. The bootstrap. Handbook of Econometrics, 5:3159-3228, 2001.

[66] E. Mammen. When does bootstrap work?: Asymptotic results and simulations. SpringerVerlag, 1992.

[67] R. Davidson and E. Flachaire. The wild bootstrap, tamed at last. Journal of Econometrics, 146(1), 2008.

[68] I. Verdinelli. Advances in bayesian experimental design (with discussion). Bayesian Statistics, 4:467-481, 1992.

[69] I. Verdinelli and J. B. Kadane. Bayesian designs for maximizing information and outcome. Journal of the American Statistical Association, 87:510-515, 1992.

[70] M. Clyde and K. Chaloner. The equivalence of constrained and weighted designs in multiple objective design problems. Journal of the American Statistical Association, 91:1236-1244, 1996.

[71] E. Zitzler, K. Deb, and L. Thiele. Comparison of multiobjective evolutionary algorithms: empirical results. Evolutionary Computation, 8:173-195, 2000.

[72] D.F. Jones, S.K. Mirrazavi, and M. Tamiz. Muiti-objective meta-heuristics: An overview of the current state-of-art. European Journal of Operational Research, 137:1-9, 2002.

[73] E. Zitzler, M. Laumanns, and L. Thiele. Spea2: Improving the strength pareto evolutionary algorithm. Swiss Federal Institute Techonology, 2001.

[74] I.H. Sloan. When are quasi-monte carlo algorithms effcient for high dimensional integrals? Journalof Complexity, 14:1-33, 1998.

[75] P. M. Boerrigter, G. Te Velde, and J. E. Baerends. Three-dimensional numerical integration for electronic structure calculations. International Journal of Quantum Chemistry, 33:88-113, 1988.

[76] B. S. Skrainka and K.L. Judd. High performance quadrature rules: How numerical integration affects a popular model of product differentiation. Working Paper. 
[77] C. R. Bhat. Quasi-random maximum simulated likelihood estimation of the mixed multinomial logit model. Transportation Research Part B: Methodological, 35:677-693, 2001.

[78] F. Heiss and V. Winschel. Likelihood approximation by numerical integration on sparse grids. Journal of Econometrics, 144:62-80, 2008.

[79] R. Cools. Advances in multidimensional integration. Journal of Computational and Applied Mathematics, 149:1-12, 2002.

[80] A.H. Stroud and D. Secrest. Gaussian quadrature formulas. Prentice-Hall, Englewood Cliffs, N.J. 